\title{
HRAS germline mutations impair LKB1/AMPK signaling and mitochondrial homeostasis in Costello syndrome models
}

\author{
Laetitia Dard,,$^{1,2,3}$ Christophe Hubert, ${ }^{1,2}$ Pauline Esteves, ${ }^{1,2}$ Wendy Blanchard, ${ }^{1,2,3}$ China Bou About, ${ }^{4}$ Lyla Baldasseroni, ${ }^{5}$ \\ Elodie Dumon, ${ }^{1,2}$ Chloe Angelini, ${ }^{1,2,6}$ Mégane Delourme, ${ }^{5}$ Véronique Guyonnet-Dupérat, ${ }^{2,7}$ Stéphane Claverol, ${ }^{2,8}$ Laura Fontenille, ${ }^{9}$ \\ Karima Kissa, ${ }^{9}$ Pierre-Emmanuel Séguéla, ${ }^{2,10,11}$ Jean-Benoît Thambo, ${ }^{2,10,11}$ Lévy Nicolas, ${ }^{5}$ Yann Herault, ${ }^{4,12}$ Nadège Bellance, ${ }^{1,2}$ \\ Nivea Dias Amoedo, ${ }^{1,2,3}$ Frédérique Magdinier, ${ }^{5}$ Tania Sorg, ${ }^{4}$ Didier Lacombe, ${ }^{1,2,6}$ and Rodrigue Rossignol ${ }^{1,2,3}$

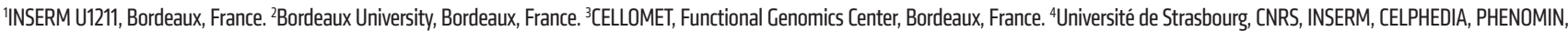 \\ Institut Clinique de la Souris, Illkirch, France. ${ }^{5}$ Aix-Marseille University, INSERM, Marseille Medical Genetics, Marseille, France. ${ }^{6}$ Medical Genetics Department, Bordeaux University Hospital, Bordeaux, France. \\ 'Vect'UB, vectorology platform, INSERM USO05 - CNRS UMS 3427-TBM-Core, and ${ }^{8}$ Plateforme Proteome, University of Bordeaux, Bordeaux, France. ${ }^{9} \mathrm{AZELEAD}$, Montpellier, France. ${ }^{10} \mathrm{CHU}$ Bordeaux, \\ Haut-Lévèque Hospital, Cardiology Department, Bordeaux, France. "IHU LYRIC, Pessac, France. "2Université de Strasbourg, CNRS, INSERM Institut de Génétique et de Biologie Moléculaire et Cellulaire, IIlkirch, France.
}

Germline mutations that activate genes in the canonical RAS/MAPK signaling pathway are responsible for rare human developmental disorders known as RASopathies. Here, we analyzed the molecular determinants of Costello syndrome (CS) using a mouse model expressing HRAS p.G12S, patient skin fibroblasts, hiPSC-derived human cardiomyocytes, a HRAS p.G12V zebrafish model, and human fibroblasts expressing lentiviral constructs carrying HRAS p.C12S or HRAS p.G12A mutations. The findings revealed alteration of mitochondrial proteostasis and defective oxidative phosphorylation in the heart and skeletal muscle of CS mice that were also found in the cell models of the disease. The underpinning mechanisms involved the inhibition of the AMPK signaling pathway by mutant forms of HRAS, leading to alteration of mitochondrial proteostasis and bioenergetics. Pharmacological activation of mitochondrial bioenergetics and quality control restored organelle function in HRAS p.G12A and p.G12S cell models, reduced left ventricle hypertrophy in CS mice, and diminished the occurrence of developmental defects in the CS zebrafish model. Collectively, these findings highlight the importance of mitochondrial proteostasis and bioenergetics in the pathophysiology of RASopathies and suggest that patients with CS may benefit from treatment with mitochondrial modulators.

\section{Introduction}

Germline mutations that activate RAS/MAPK signaling are responsible for the "RASopathies," a group of rare human developmental diseases that affect more than 400,000 individuals in the United States alone. Costello syndrome (CS) has been described as a multiple congenital anomaly syndrome caused by heterozygous activating germline mutations in HRAS (1). Most individuals affected by CS carry a mutation in HRAS at the G12 position, and more than $80 \%$ of individuals with CS have a p.G12S substitution. Most children with CS exhibit an increased birth weight, dysmorphic craniofacial features, failure to thrive, and gastroesophageal reflux with oral aversion, especially during the newborn period. CS can also involve the skin, with excessive wrinkling and redun-

\section{Related Commentary: https://doi.org/10.1172/JCl157560}

Conflict of interest: RR, DL, LD, and NDA report a patent (PCT/EP2022/051382) on the use of the bezafibrate and urolithin A pharmacological drug combination for the treatment of hypertrophic cardiomyopathy in Costello syndrome.

Copyright: () 2022, Dard et al. This is an open access article published under the terms of the Creative Commons Attribution 4.0 International License.

Submitted: June 13, 2019; Accepted: February 23, 2022; Published: April 15, 2022. Reference information: J Clin Invest. 2022;132(8):e131053.

https://doi.org/10.1172/JCl131053. dancy over the dorsum of the hands and feet, along with deep plantar and palmar creases, as well as an increased risk of developing benign or malignant tumors.

A central feature of CS and other RASopathies is hypertrophic cardiomyopathy (HCM; refs. 2, 3). Musculoskeletal abnormalities such as hypotonia have also been reported in CS (4). Furthermore, HCM was observed in transgenic mutant Hras mouse models and in patients expressing constitutively active forms of HRAS (5-10); however, the molecular mechanisms linking HRAS activation with cardiac dysfunction remain unknown. However, cardiac involvement remains a major determinant for the prognosis of CS, raising the need to identify the molecular causes of HCM and propose adapted therapeutic strategies. Several lines of evidence have indicated that HCM typically associates with energetic insufficiency caused by bioenergetic alterations of the heart (11-13). According to the American Heart Association, "alterations in mitochondrial function are increasingly being recognized as a contributing factor in myocardial infarction and in patients presenting with cardiomyopathy" (14). Therefore, we hypothesized that CS could include early alterations of heart bioenergetics participating in the development of HCM. Our hypothesis was also based on a prospective screening of 18 patients with various RAS/MAPK pathway defects that detected biochemical signs of disturbed oxidative phosphor- 
ylation (OXPHOS) (15) as well as on the partial overlap of clinical manifestations between mitochondrial diseases and RASopathies (16). The central role of mitochondrial dysfunction in HCM pathophysiology was also demonstrated in vivo using transgenic mice, with the following KOs: Prkaa2 (17), Ppargc1a (18, 19), Nfe2l2 (20), Esrra (21), Ppara (22), Clpp (23) or Yme1l1 (24).

In the present study, we explored the bioenergetics of the heart and skeletal muscle in different cell and animal models of CS and discovered alteration in the molecular control of mitochondrial proteostasis. We tested the effect of mitochondrial turnover and bioenergetics pharmacological stimulation on HCM prevention at the preclinical stage in the CS mouse and zebrafish models.

\section{Results}

Patients with CS and a mouse model exhibit left ventricular HCM. First, we regrouped a cohort of 10 patients with CS (Supplemental Table 1; supplemental material available online with this article; https://doi.org/10.1172/JCI131053DS1) to investigate heart structure and function. Echocardiography revealed a characteristic left ventricular hypertrophy in $70 \%$ of the patients (Supplemental Figure 1, A-D). Electrocardiograms obtained of patients with CS further revealed that $50 \%$ of the patients had abnormal deviation of the heart axis (Supplemental Table 2). Two patients had a right deviation of the electrical axis with discrete echocardiographic left ventricular hypertrophy. Furthermore, pathologic Q waves were present in $40 \%$ of patients, and all patients with pathologic Q waves had only discrete ventricular hypertrophy on echocardiography. In the heterozygous HRAS p.G12S mouse model of CS, we observed increased left ventricle volume (Figure 1, A-F, and Supplemental Figure 1, F and G) and cardiomegaly (Supplemental Figure 1E and Supplemental Figure 2). A significant increase in systolic arterial blood pressure was also observed in these animals at 8 and 20 weeks of age (Supplemental Figure 1H) without alteration in heart beating rate (Supplemental Figure 1I). Likewise, hypertension was observed in all the patients with CS considered in this study (Supplemental Table 1). Further evaluation of the heart structure and function by echocardiography confirmed the increase in left ventricle volume at diastole and systole (Figure $1, \mathrm{E}$ and $\mathrm{F}$ ). Evaluation of the left ventricular diastolic function, including early and late mitral flow $\mathrm{E}$ wave velocities revealed a significant increase in these 2 parameters in 23-week-old CS mice (Figure 1, I-K). These findings might reflect increased left atrial filling pressures in the left atrium. The $\mathrm{E}$ wave velocity through the tricuspid valve was also increased (Figure 1, L-N), suggesting occurrence of tricuspid regurgitation. Tricuspid regurgitation, in combination with left ventricular dysfunction was associated with excess mortality in previous studies (25). Last, the pulmonary vein peak velocity was also reduced in 23-week-old CS mice (Figure $1 G)$. Comparison of the echocardiography studies performed at 11 and 23 weeks of age showed that the cardiac disease was progressive and did not reach yet the state of heart failure, as the ejection fraction and the $\mathrm{E} / \mathrm{A}$ ratio remained unaltered (Figure $1, \mathrm{H}$ and $\mathrm{K}$, and Supplemental Figure 3). Nevertheless, the descending aorta peak velocity was significantly reduced in the CS mouse heart (Figure 1, $\mathrm{O}$ and $\mathrm{P}$ ), and the establishment of a ventricular dilation (Figure 1, A and F) with altered mitral and tricuspid flow $\mathrm{E}$ wave velocities (Figure 1, I, J, and $\mathrm{L}-\mathrm{N}$ ) was significant in the CS mouse model. These observations indicate that left ventricular cardiac hypertrophy is a characteristic feature of CS in most patients and in the HRAS p.G12S mouse model.

The CS mouse model exhibits heart and skeletal muscle bioenergetic dysfunction. Mitochondrial dysfunction is an important determinant of HCM (26); thus, we investigated heart bioenergetics in the CS mouse model. The in situ evaluation of OXPHOS (Figure 2A) was performed in heart-permeabilized muscle fibers obtained from control or heterozygous 12-week-old CS mice. This analysis revealed a reduced rate of coupled respiration (state 3 ) associated with a decreased rate of ATP synthesis (Figure 2, B-D). Measurement of the respiratory chain complex enzymatic activity (Figure 2, $\mathrm{E}$ and $\mathrm{F}$ ) demonstrated a generalized decrease in complex I-IV activity in the CS mouse heart (Figure 2E). Respiratory chain complex enzymatic activity defects were also discovered in the skeletal muscle of CS mice (Figure 2F). Furthermore, histo-enzymology determination of complex IV activity in the skeletal muscle showed a strong reduction in the number of COX-positive fibers (Figure 2, G and $\mathrm{H}$ ). Accordingly, a reduction of state 3 respiration and mitochondrial ATP synthesis were observed in permeabilized skeletal muscle fibers obtained from 12-week-old heterozygous CS mice (Supplemental Figure 3). These findings revealed the existence of mitochondrial dysfunction in both the myocardium and skeletal muscle of CS mice.

Mitochondrial proteostasis is altered in CS. To unravel the molecular mechanisms underpinning early OXPHOS dysfunction in the CS mouse model we performed a label-free proteomic analysis (Figure 3A) of (a) 4 CS mouse tissues (skeletal muscle, heart, liver, and brain) obtained at 3 and 12 weeks of age from homo- and heterozygous HRAS p.G12S animals, (b) human primary skin fibroblasts obtained from 3 patients with CS carrying HRAS p.G12S or HRAS p.G12A mutations, and (c) human primary skin fibroblasts expressing ectopic HRAS p.G12S or HRAS p.G12A lentiviral constructs ( $n=4$ clones for each construct). Comprehensive analysis of the cellular biochemical processes altered by mutant HRAS (Figure 3B) consistently identified "mitochondrial dysfunction" and "OXPHOS" with the highest score $(-\log (P$ value $))$ in each of the CS models, suggesting a generalized alteration of the mitochondrial proteome homeostasis consecutive to germline and somatic HRAS mutation. However, the direction of the changes observed in the content of selected mitochondrial proteins varied between tissues. For instance, a predominant decrease of OXPHOS components was detected in the heart and liver, while a dominating accumulation of these proteins was observed in the muscle, brain, and skin fibroblasts from patients with CS as well as in the related mutated HRAS p.G12S- and p.G12A-expressing cell models (Figure $3 \mathrm{C}$ ). A focus on the common molecular changes that occurred in the hearts and livers, 2 tissues with very different functions, of 3-week-old heterozygous and homozygous CS mice revealed a reduction of fatty acid oxidation enzymes and $5^{\prime}$ AMP-activated protein kinase (AMPK) indirect transcriptomic targets (Figure 3, D and E). These findings raised the hypothesis that HRAS p.G12S mutation could trigger alteration of mitochondrial proteome homeostasis with a more pronounced effect on mitochondrial biogenesis or degradation according to the tissue considered and stage of development. Given the role of the LKB1/ AMPK signaling axis in the tissue-specific control of mitochondri- 

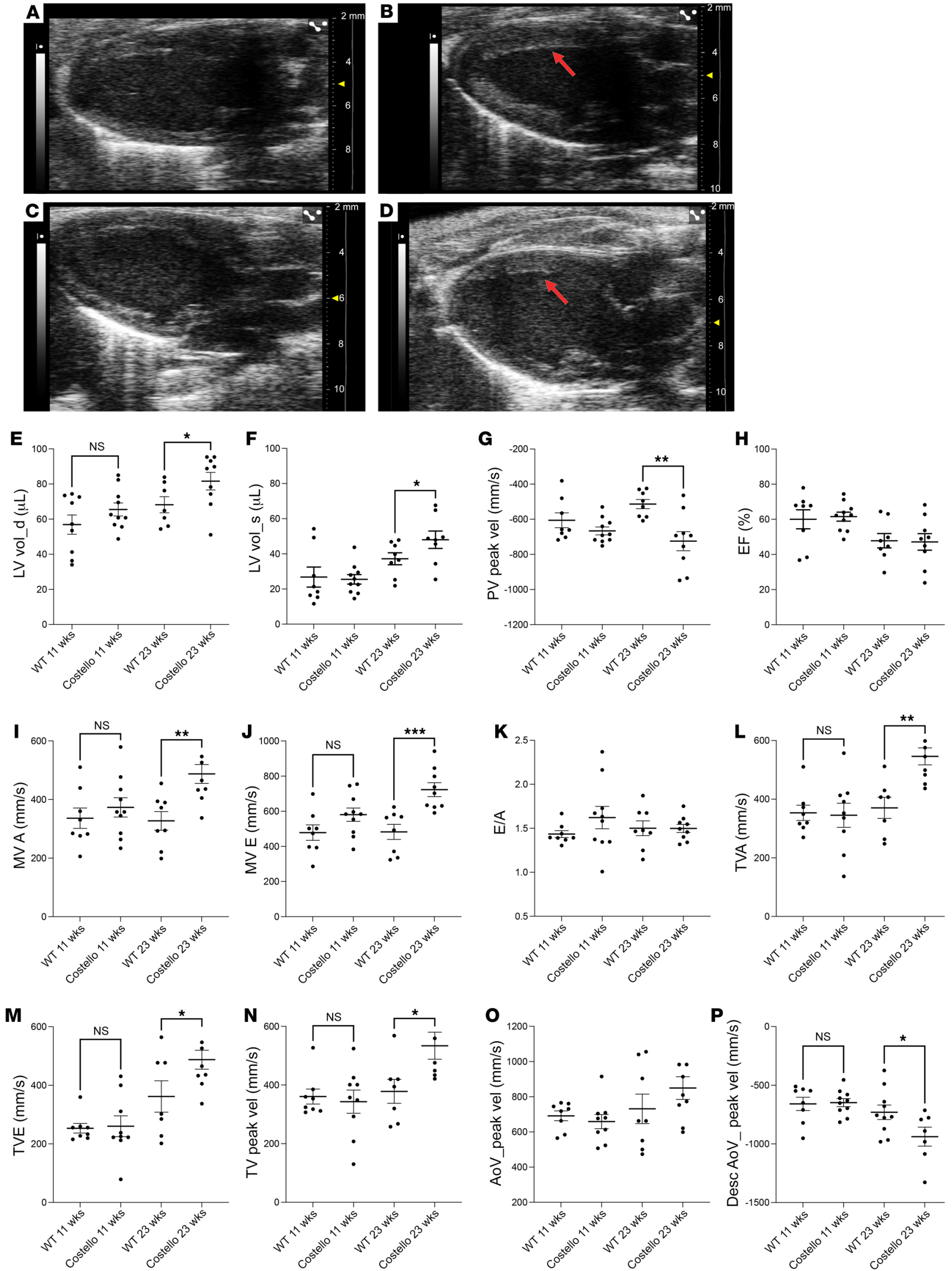
Figure 1. Hypertrophic cardiomyopathy in patients with CS and the mouse model. Parasternal echography was performed in (A and C) WT (HRAS) or (B and D) Costello (HRAS p.G12S) mice at 11 weeks (A and B) and 23 weeks ( $\mathbf{C}$ and $\mathbf{D}$ ) of age. Left ventricle hypertrophy is indicated by arrows. (E) Left ventricle volume at diastole (LV vol_d), (F) left ventricle volume at systole (LV vol_s), (G) pulmonary valve peak velocity (PV peak vel), (H) ejection fraction (EF), (I) mitral valve flow A wave maximal velocity (MV A), (J) mitral valve flow $E$ wave maximal velocity (MV E), (K) E/A ratio, (L) tricuspid valve flow A wave maximal velocity (TVA), (M) tricuspid valve flow $\mathrm{E}$ wave maximal velocity (TVE), (N) tricuspid valve peak velocity (TV peak vel), (0) aorta peak velocity (AoV_peak vel), and (P) descending aorta peak velocity (Desc AoV_peak vel). The data are expressed as the mean \pm SEM. ${ }^{*} P<0.05,{ }^{* *} P<0.01,{ }^{* * *} P<0.001$ (unpaired $t$ test was performed between the CS mouse group and the corresponding age-matched WT animals). HCM, hypertrophic cardiomyopathy.

al biogenesis and degradation, we hypothesized that altered LKB1 and/or AMPK function could be a consequence of HRAS hyperactivation in CS (Figure 3F).

HRAS hyperactivation inhibits the LKB1/AMPK pathway. Investigation of the bioenergetic changes in skin fibroblasts from patients with CS and ectopic HRAS p.G12A or HRAS p.G12Sexpressing human skin fibroblasts revealed a reduction of OXPHOS function and capacity (Figure 4, A and B) associated with alteration of respiratory chain proteostasis (Figure 3, B and C). In line with the hypothesis of a deregulated LKB1/AMPK signaling axis, we first determined the global content of AMPK $\alpha 1$ and $\alpha 2$ subunits by Western blot using a polyclonal antibody that could not distinguish between the 2 subunits (Figure 4, C-E). The results showed a global decrease in AMPK $\alpha 1+\alpha 2$ subunits in CS cell models, cells from patients with CS, and human skin fibroblasts expressing mutant HRAS p.G12A or HRAS p.G12S. To add precision to our findings, we performed Q-PCR investigations to detect the specific changes in PRKAA1 or PRKAA2 subunits (Figure 4F). The results showed a significant downregulation (-75\%) of the PRKAA2 subunit mRNA content in cells from patients with CS and mutant HRAS p.G12A and p.G12S cell models but also 12-week-old HRAS p.G12S heterozygous CS mouse hearts. As AMPK $\alpha 2$ mediates AMPK activation via threonine 172 phosphorylation, a corresponding reduced expression of the pAMPK (thr172) content was detected in the different CS cell models (Figure 4, C-E). Our findings demonstrated that mutant HRAS p.G12A and p.G12S stimulates HRAS activation (Figure 4, $\mathrm{G}$ and $\mathrm{H}$ ) and inhibits, in turn, the expression of the AMPK $\alpha 2$ subunit that is essential for AMPK activation. Moreover, analysis of the LKB1 phospho-activation status, as determined by the phosphorylation level of serine 428 , revealed a strong reduction in cells from patients with CS. Finally, the phosphorylation level of ACC, a canonical target of AMPK was reduced in patient skin fibroblasts, although the level of ACC phosphorylation was low in our experimental conditions (Supplemental Figure 4). Therefore, the LKB1/AMPK signaling axis was inhibited at 2 levels in fibroblasts from patients with CS (Figure 4I). Similar findings were obtained in 3-week-old CS mouse hearts, as the AMPK $\alpha 1+\alpha 2$ and LKB1 protein content were substantially decreased (Figure 4J). In cells from patients with CS, the observed accumulation of mitochondrial proteins (Figure 3C) suggested a predominant alteration of mitochondrial proteostasis at the level of mitochondrial degradation, a complex process dependent on autophagy-mediated bulk degradation of mitochondria (mitophagy), and protease-mediated specific degradation of mitochondrial proteins. Accordingly, comparative proteomics revealed a marked reduction of the autophagy regulator ATG7 and of the mitochondrial protease CLPx (Figure 4K), 2 elements essential for mitochondrial clearance. Further evaluation of the mechanisms involved in mitochondrial proteostasis was performed in the CS mouse heart (Supplemental Figure 5) and skin fibroblasts from patients with CS (Supplemental Figure 6). This analysis revealed that AMPK, CRIF1, and SQSTM1/p62 were consistently reduced both in CS mouse heart and in skin fibroblasts from patients with CS. Previous work has already shown that AMPK, CLPX, ATG7, CRIF1, and SQSTM1/p62 inhibition trigger defective mitochondrial proteostasis, cellular bioenergetics impairment, and inhibition of the autophagic flux (27-30). Accordingly we measured a defective mitochondrial bioenergetics (Figure 2, B-D) and decreased autophagic flux (Figure 4, L-N) in the different CS cell models. The mitochondrial proteostasis regulator CLPX, which was downregulated in CS cells, had previously been identified as a transcriptomic target of AMPK and PGC1 $\alpha(31,32), 2$ large-scale transcriptional regulators strongly downregulated in CS cells.

Mitochondrial biogenesis, the second arm of mitochondrial turnover controlled by AMPK, was evaluated by measuring the expression level of TFAM in cells from patients with CS using Q-PCR (Figure 4O). TFAM content was decreased in cells from patients with CS (as well as in CS mouse heart; Supplemental Figure 5), in line with an alteration of mitochondrial biogenesis. These findings indicate that the LKB1/AMPK signaling axis is altered in cells from patients with CS and CS mouse hearts and that mitochondrial biogenesis and degradation processes are also impaired in these cells. However, the proteomic investigation of CS mouse hearts revealed additional changes in proteins specifically involved in the pathophysiology of cardiomyopathy (Supplemental Figure 7), such as CRYAB, TNN or MYBPC3, suggesting that LKB1/AMPK signaling deregulation is only one mechanism contributing to the complexity of CS heart pathophysiology, as expected in a condition of HRAS/MAPK pathway overactivation. Moreover, comparative analysis of 4 CS mouse tissues taken at 3 weeks of age suggested that alteration of mitochondrial proteostasis in CS mice is tissue specific (Supplemental Figure 8).

miR-221* inhibits AMPKa 2 expression in mutant HRAS-expressing cells. Parcellar evidence suggested the existence of a RAS/ miR-221*/AMPK regulatory axis in human cancer cells (33-36). Moreover, miR-221* overexpression was observed in patients with cardiac hypertrophy after sudden death (37), as can occur in patients with CS. Thus, we evaluated the implication of this pathway in CS pathophysiology using different approaches. First, in silico analysis of the differential proteomics data obtained in skin fibroblasts from patients with CS and in mutant HRAS-expressing cells showed that a common core of 71 proteins downregulated by germline HRAS mutation were predicted targets of miR$221^{*}$ (Supplemental Figure 9, A and B). These proteins play a role in cellular functions, such as cell migration, cell proliferation, and cell adhesion (Supplemental Figure 9, C and D). Moreover, miR$221^{*}$ expression was increased in fibroblasts from patients with CS and in human fibroblasts expressing ectopic mutant forms of 
A
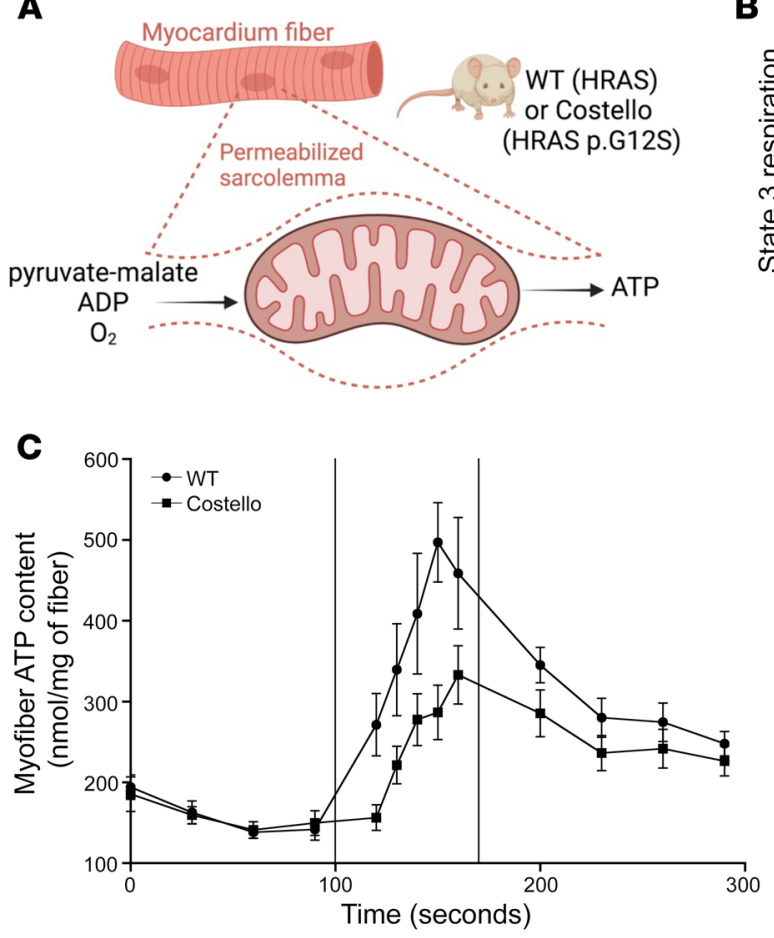

E

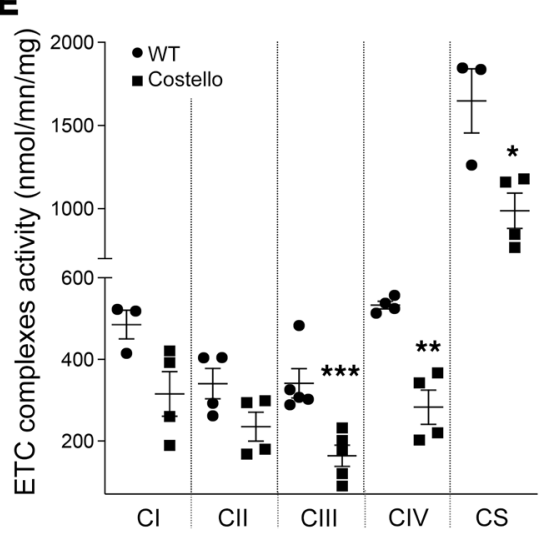

B
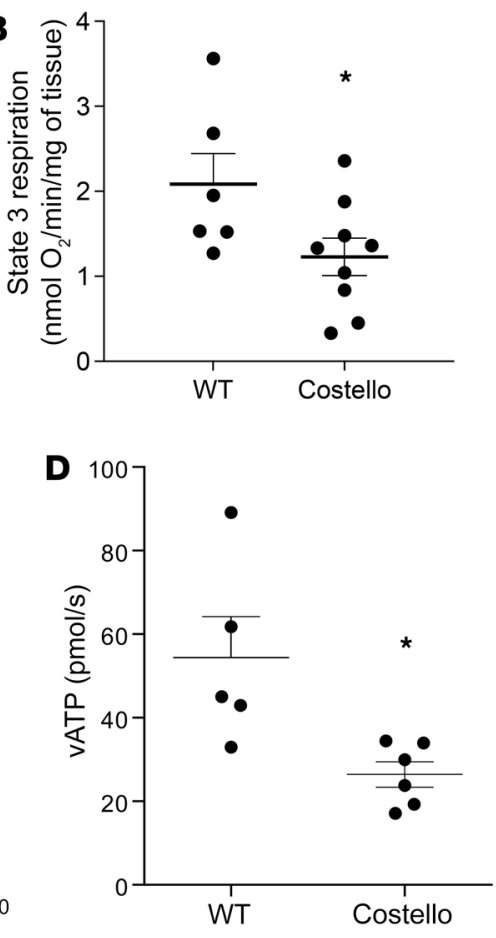

Figure 2. Mitochondrial bioenergetics is altered in situ in the CS mouse heart and skeletal muscle. (A) Permeabi-

lized heart muscle fibers bioenergetics evaluation methods. (B) Rate of coupled (ADP-stimulated "state 3") respiration was determined in situ using highresolution respirometry (WT, $n=6$; Costello, $n=9$ ). (C and $\mathbf{D}$ ) Rate of mitochondrial ATP synthesis (vATp) determined in heart-permeabilized muscle fibers (WT, $n=5$; Costello, $n=6$ ). (E) Electron transport chain (ETC) complex enzymatic activities determined in WT or Costello mouse model hearts (WT, $n=$ 4; Costello, $n=4$ ). (F) Respiratory chain complex enzymatic activities determined in WT or Costello mouse model skeletal muscle (WT, $n=4$; Costello, $n$ =4). (C) Histo-enzymology staining of the respiratory chain complex IV (COX) specific activity. Muscle fibers and their nuclei were stained using $\mathrm{H} \& \mathrm{E}$. Original magnification, $\times 1000$. (H) Quantification of the COX-positive muscle fibers (WT, $n=3$; Costello, $n=3$ ). Data are expressed as the mean $\pm \mathrm{SEM} .{ }^{*} P<0.05,{ }^{* *} P<$ $0.01,{ }^{* * *} P<0.001$ (unpaired $t$ test).
G

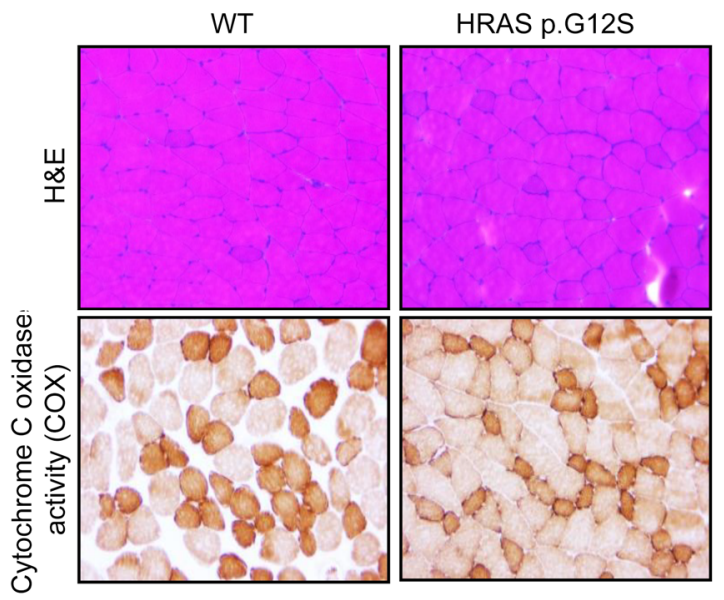

F

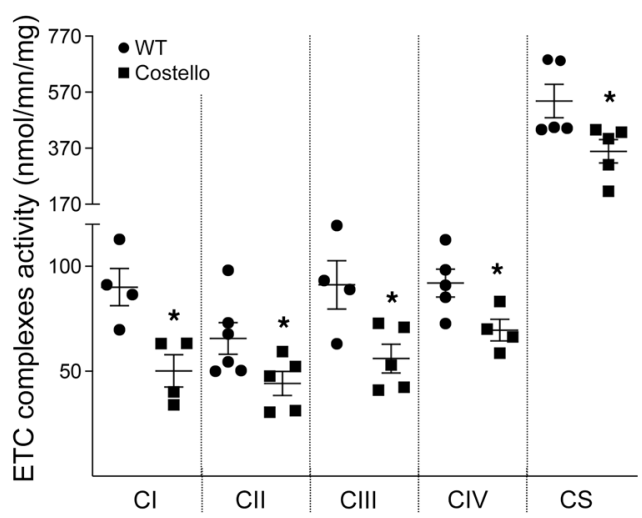

H

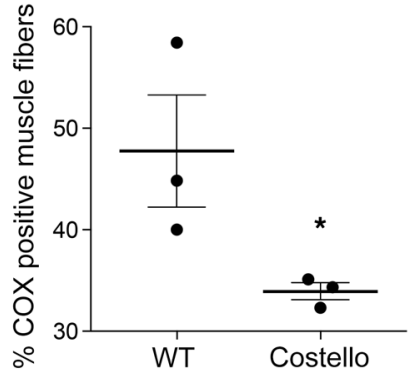


A
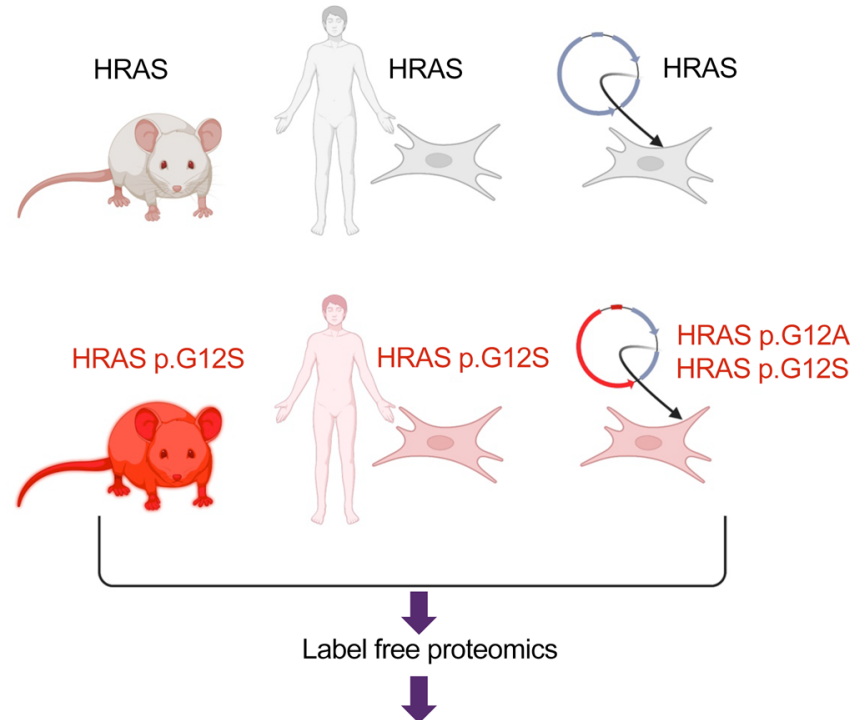

Pathway analysis (Costello/WT)

B
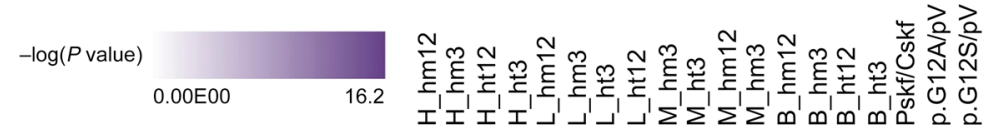

Mitochondrial dysfunction

EIF2 signaling

Oxidative phosphorylation

Sirtuin signaling pathway

Regulation of elF4 and p70S6K signaling

Protein ubiquitination pathway

TCA cycle II (eukaryotic)

Remodeling of epithelial adherens junctions

mTOR signaling

Actin cytoskeleton signaling

Glycolsis I

Integrin signaling

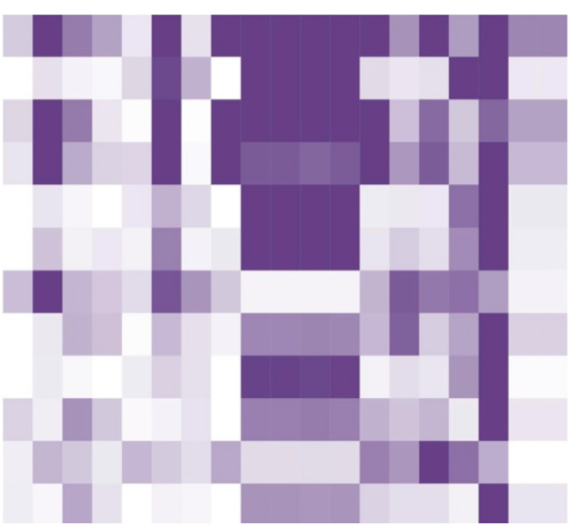

D Fatty acid oxidation

E AMPK transcriptomic targets
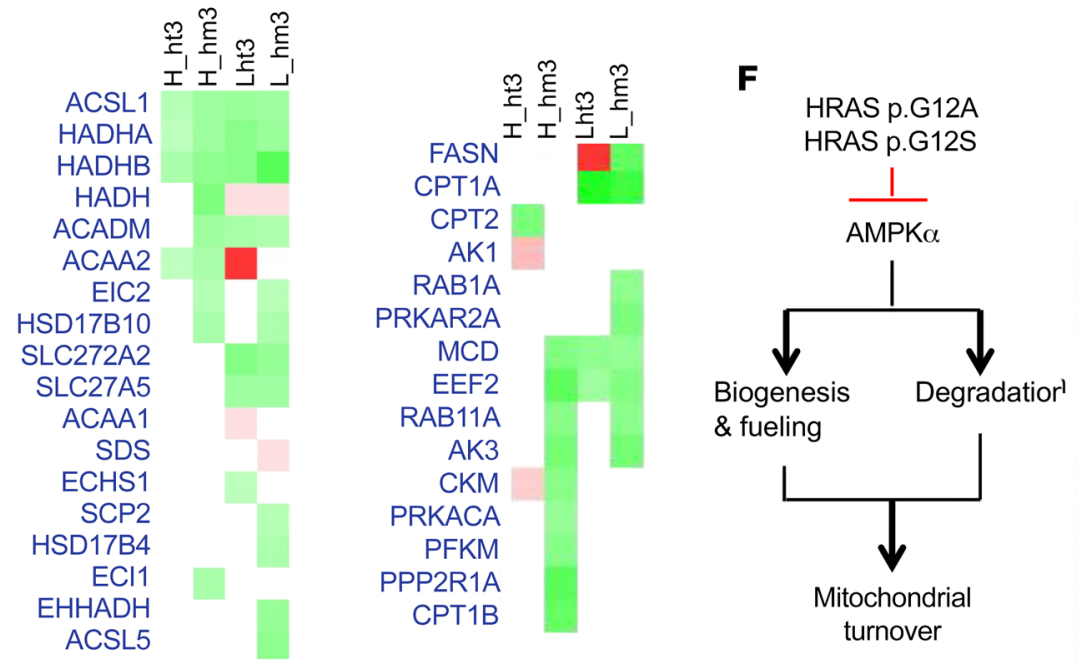

$-\log (P$ value $)$
$\begin{array}{cc}0.00 E 00 \\ \text { Expr fold change }\left.\right|_{-6069.644} & 1.80 \mathrm{E} 308\end{array}$ $\begin{gathered}\text { Mitochondrial } \\ \text { dysfunction }\end{gathered}$

Genes in the

mitochondria

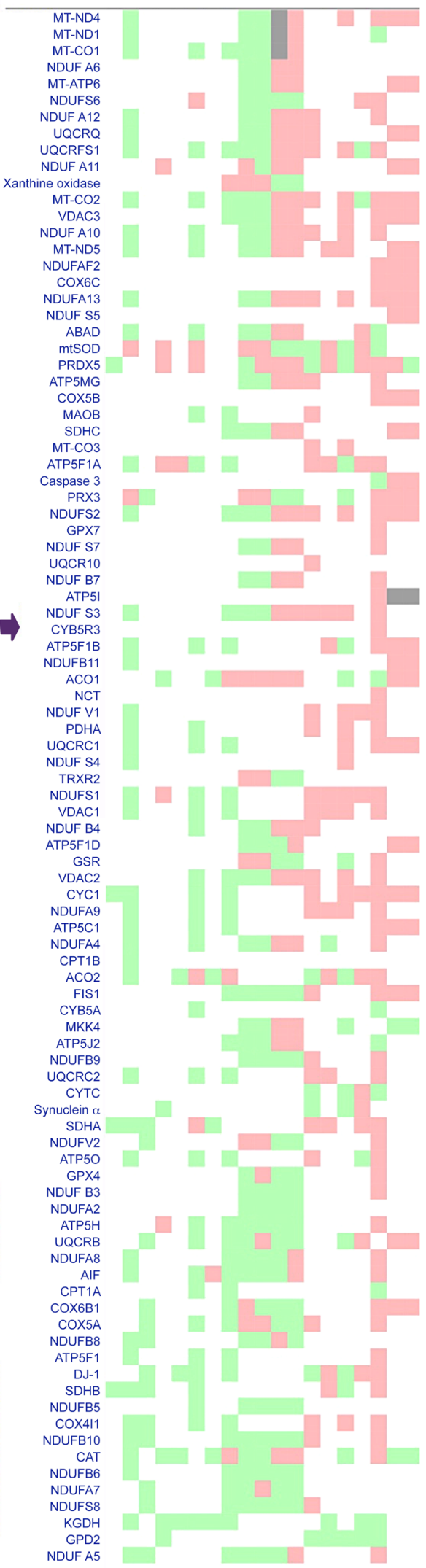


Figure 3. Mitochondrial proteostasis is compromised in Costello syndrome. (A) Description of the 3 types of Costello syndrome (CS) models used in the label-free proteomic analysis. A differential proteome was obtained between WT and CS models. Ingenuity Pathway Analysis (Qiagen; version 01-07) was used to perform a comparative analysis of the data. $n=3$ was used for each sample. (B) Top canonical pathways altered in the $[S$ biological models [ranked by $-\log (P$ value $)$. (C) Detail of the changes in the "mitochondrial dysfunction" category (expression fold change; green, downregulation; red, upregulation). (D and E) Changes in the fatty acid oxidation and AMPK signaling (indirect transcriptomic targets) proteins detected in the hearts and livers of 3-week-old CS mice (heterozygous or homozygous HRAS p.G12S) compared with WT mice. (F) Working hypothesis on the effect of mutant HRAS p.G12A and HRAS p.G12S on mitochondrial biogenesis and degradation via inhibition of AMPK signaling. $\mathrm{H}$, heart; $\mathrm{B}$, brain; L, liver; $\mathrm{M}$, skeletal muscle; ht, heterozygous; hm, homozygous; 3, 3-week-old mice; 12, 12-week-old mice; Pskf, patient skin fibroblasts; Cskf, control skin fibroblasts; p.G12A, human skin fibroblasts transduced with HRAS p.G12A-expressing lentiviral plasmid; p.G12S, human skin fibroblasts transduced with HRAS p.G12S-expressing lentiviral plasmid; $\mathrm{pV}$, human skin fibroblasts transduced with empty lentiviral plasmid.

HRAS (Figure 5A), in agreement with our hypothesis (Figure 5B). Furthermore, inhibition of miR-221* expression using a specific anti-miR rescued the expression of AMPK $\alpha 2$ protein, $\mathrm{mRNA}$, and activity in HRAS p.G12S- or HRAS p.G12A-expressing cells (Figure $5, \mathrm{C}-\mathrm{E}$ ). The rescuing effect of anti-miR-221* was also found at the level of AMPK and PGC1 $\alpha$ expression, mitochondrial respiration, and mitochondrial ATP levels in both cells from patients with CS and mutant HRAS cell models (Figure 5, E-G). A different sensitivity to the anti-miR-221* was observed between the HRAS p.G12S- and the HRAS p.G12A-expressing fibroblasts (Figure $5 \mathrm{C})$. In the former cell line the increase in total AMPK content was strong, while in the latter it was weak. Still, in the 2 cell lines expressing mutant forms of HRAS, the anti-miR-221* triggered a marked increase in the ${ }_{\text {thr172 }} \mathrm{P}-\mathrm{AMPK} /$ total AMPK ratio. In contrast, the miR- $221^{*}$-mimic altered cell respiration in control fibroblasts. The number of mitochondrial particles was also increased in cells from patients with CS and in mutant HRAS cell models treated with the anti-miR-221* (Figure 5, $\mathrm{H}$ and I). Finally, antimiR-221* stimulated cell growth in glucose-deprived media that forced cells to rely on OXPHOS (38) for survival (Supplemental Figure 10). Taken together, these findings indicate that HRAS p.G12A and p.G12S activate the expression of miR-221* but also that miR-221* inhibits the expression and function of AMPK $\alpha 2$ in skin fibroblasts derived from patients with CS and related cellular models. Our findings also demonstrate that both AMPK phosphorylation status (thr172) and mitochondrial biogenesis can be rescued in vitro using an anti-miR-221*. However, we could not detect the endogenous level of miR-221* in the WT and the CS mouse heart, indicating that inhibition of AMPK $\alpha 2$ by miR-221* may occur by different mechanisms in this tissue. The reduced expression of LKB1 found in the CS mouse heart (Figure 4J), as also observed in skin fibroblasts from patients with CS (Figure $4 \mathrm{I}$ ), provides an additional mechanism responsible for the inhibition of LKB1/AMPK signaling in CS.

Human induced pluripotent stem cell-derived CS cardiomyocytes have reduced ${ }_{\text {ser } 428} P-L K B 1$ and $A M P K \alpha 2$ content and bioenergetic impairment. Human induced pluripotent stem cell-derived cardiomyocytes (hiPSC-CMs) were produced from primary skin fibroblasts from 2 patients with CS and from 2 controls (Figure 6A and Supplemental Figure 11). Determination of the percentage of troponin T-positive cells (Figure 6, B and C) at day 30 after differentiation showed $72.55 \% \pm 12 \%$ of troponin-positive cells for the AG08H control clone, $55.88 \% \pm 15.6 \%$ for the AG161 control clone, $77.89 \% \pm 2.3 \%$ for the CS HRAS p.G12A clone, and $86.75 \%$ $\pm 8.7 \%$ for the HRAS p.G12S (Figure 6D). Expression of the keratin sulfate antigens Tra1-60 and Tra1-81 and the glycolipid antigen SSEA 4 was verified by flow cytometry in the 4 cell lines (Figure $6 \mathrm{E}$ ). The results showed a high expression (>95\%) of these human pluripotent stem cell markers in the different cell lines. Embryoid body formation and characterization were also performed as detailed in Methods (Supplemental Figure 12). Then, we analyzed the markers of defective mitochondrial homeostasis previously identified in CS mouse tissues, skin fibroblasts from patients with CS, and CS cell models. Accordingly, PRKAA2 expression was strongly reduced in CS hiPSC-CMs (Figure 6F), and mitochondrial ATP synthesis was also impaired (Figure 6G). More precisely, in CS hiPSC-CMs the cellular total ATP level was either not altered by the OXPHOS inhibition (HRAS p.G12A) or even increased by a glycolytic compensatory response (HRAS p.G12S). These data indicate that most of the ATP produced in CS hiPSC-CMs was obtained from glycolysis, as OXPHOS inhibition with oligomycin, antimycin $\mathrm{A}$, and rotenone did not entail a drop in the cellular ATP level. As a result, CS hiPSC-CM viability was reduced in a strictly oxidative cell growth medium (Figure $6 \mathrm{H}$ ). Finally, we observed a reduction of respiratory chain protein content in these cells (Figure 6I) as well as a decreased ${ }_{\text {ser428 }}$ P-LKB1 content (Figure 6J). No difference in AMPK $\alpha 1+2$ was detected between the CS cardiomyocytes and the WT hiPSC-CMs, and the level of ${ }_{\text {thrr172 }} \mathrm{P}-\mathrm{AMPK}$ was very low in the 2 groups. These findings indicate that the defective mitochondrial bioenergetics phenotype discovered in CS mouse heart and skin fibroblasts from patients with CS was also present in human cardiomyocytes derived from hiPSCs from patients with CS.

Mitochondrial proteostasis modulators restore organelle homeostasis in CS cell and animal models. The above-described findings suggested the evaluation of a preclinical therapeutic strategy in CS based on the stimulation of mitochondrial protein biogenesis and quality control. Bezafibrate (BZ) was selected to reach this goal, because this drug could stimulate mitochondrial bioenergetics in previous studies (39-44). Accordingly, we observed that $\mathrm{BZ}$ increased the expression of the PGC1 $\alpha 100 \mathrm{KDa}$ form, raised the level of TFAM, and reduced activation of the mTOR pathway (Figure 7A). The enzymatic activity of respiratory chain complexes was also increased by BZ treatment. Proteomics further demonstrated that $\mathrm{BZ}$ normalized the level of the autophagy regulator ATG4B but also that of ATG7, ATG3, PARK7, and TOM22 in cells from patients with CS (Supplemental Figure 13). The content of various respiratory chain subunits was also increased by BZ treatment while accumulated subunits were reduced (Supplemental Figure 13). The protein content of mitochondrial protease CLPX was also rescued by the $\mathrm{BZ}$ treatment (Figure $7 \mathrm{~B}$ ). The measurement of mitochondrial respiratory fluxes, transmembrane electric potential $(\Delta \Psi)$, as well as sensitivity to the uncoupler CCCP and OXPHOS coupling efficiency confirmed that 48-hour treatment 
A

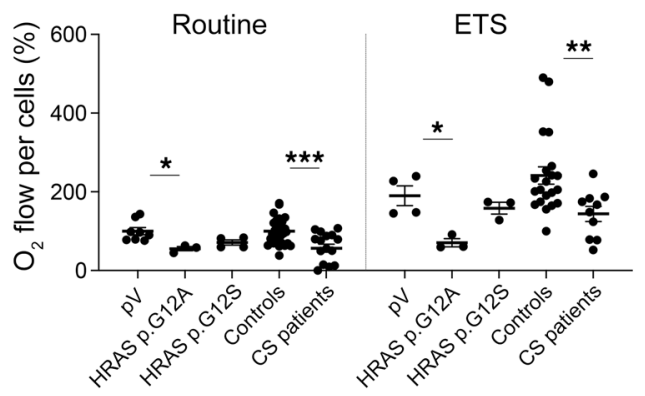

C

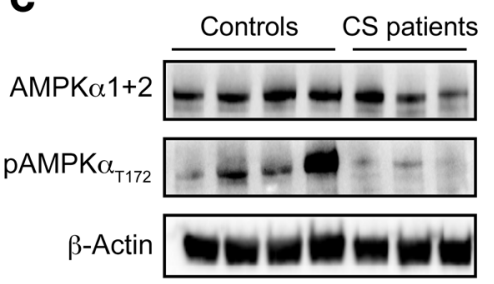

D
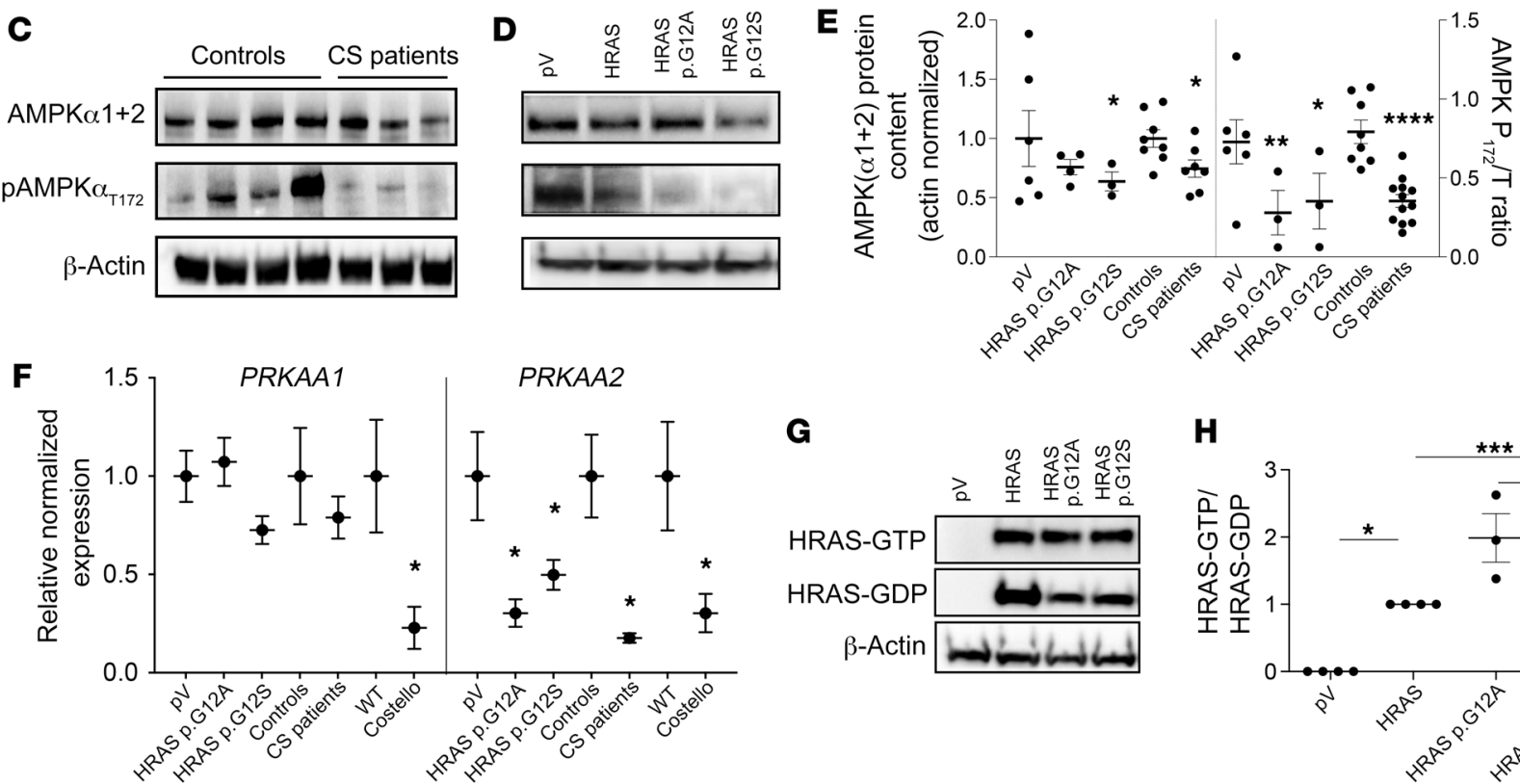

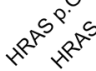
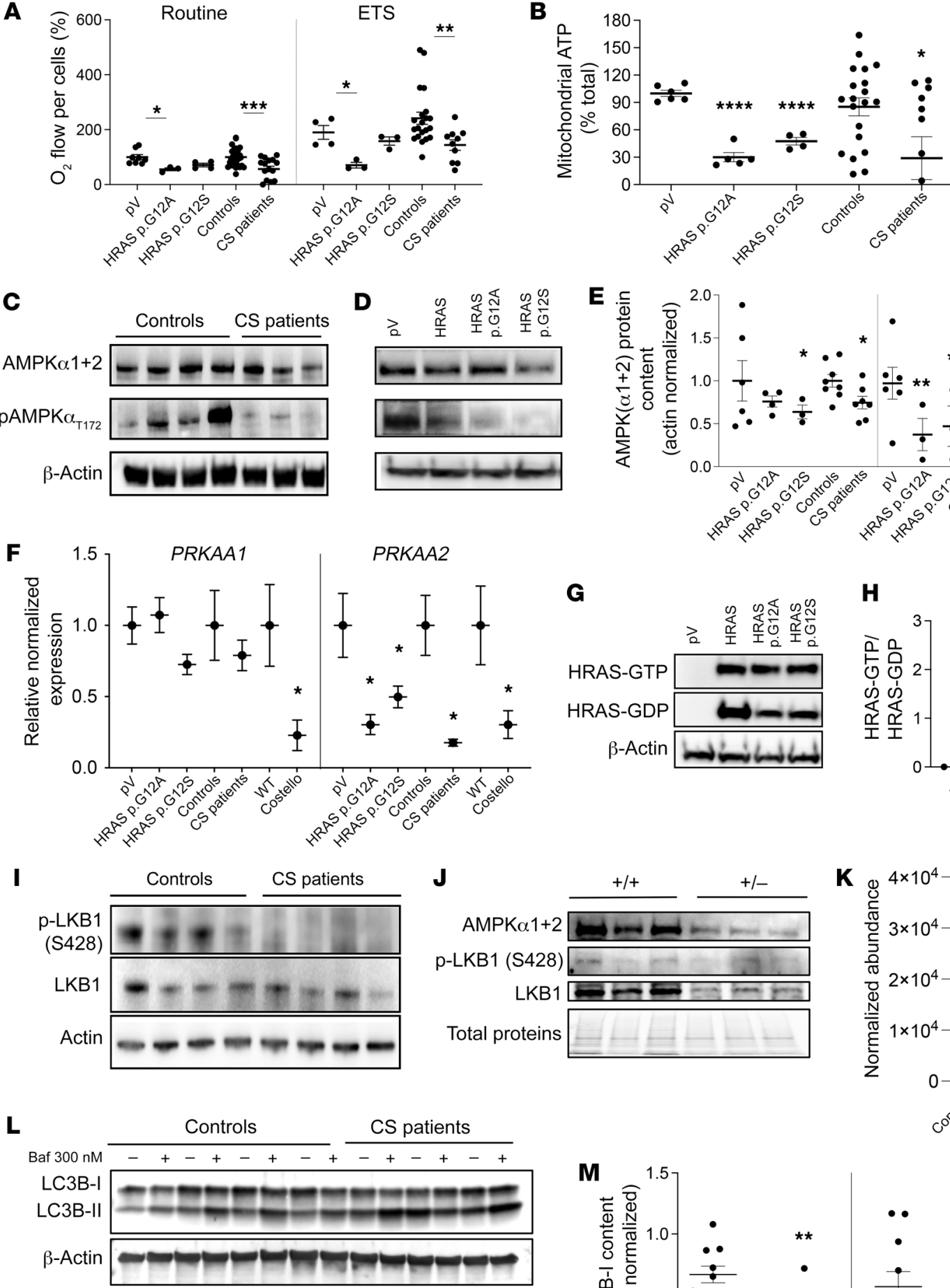

$\mathbf{L}$

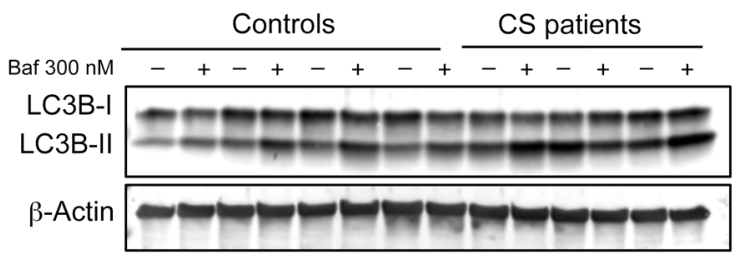

$\mathbf{N}$

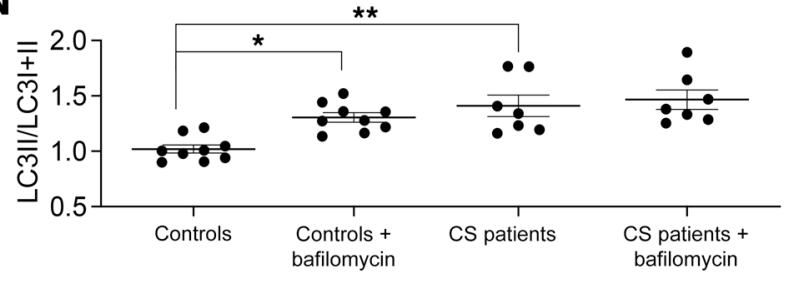

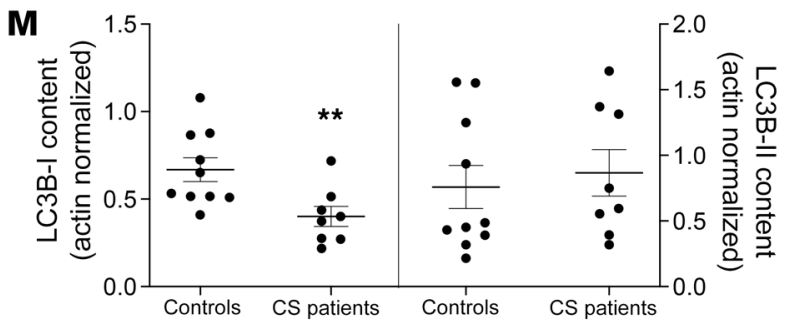

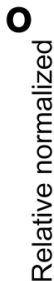

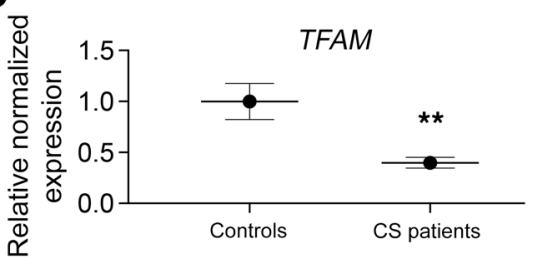

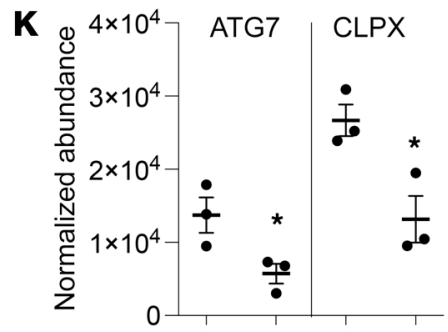

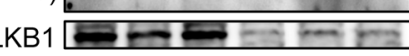

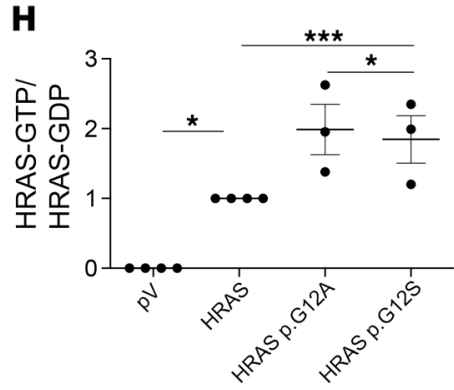


Figure 4. AMPKa2 expression is inhibited by mutant HRAS. (A) Mitochondrial respiration determined in skin fibroblasts from patients with CS and in WT human fibroblasts transduced with an empty plasmid (pV) or mutant forms of HRAS ( $n \geq 3$ for each condition). (B) Mitochondrial ATP content (expressed as a percentage of the total ATP) ( $n \geq 3)$. (C) Total AMPK $\alpha$ subunits $(\alpha 1+\alpha 2)$ and T172_phospho-AMPK protein content were quantified in cells from patients with CS $(n=6)$ and WT controls $(n=6)$. (Phospho-AMPK/[phospho-AMPK+total AMPK]) was denominated "AMPK $P_{172} / T$ ratio." ( $\boldsymbol{D}$ and $\left.\mathbf{E}\right)$ AMPK and T172_phospho-AMPK protein content determined in mutant HRAS-expressing human skin fibroblasts ( $p V$, empty plasmid; HRAS, WT gene; HRAS p.G12A and HRAS p.G12S, mutated forms of the gene) ( $n \geq 3$ ). (F) mRNA content of 2 AMPK subunits ( $\alpha 1$ and $\alpha 2)$ in different CS models: CS mouse heart, fibroblasts from patients with CS, and mutant HRAS cell models $(n=3)$. ( $(\mathbf{G}$ and $\mathbf{H}$ ) Effect of HRAS mutations on HRAS activity $(n=3)$. (I) LKB1 expression level and its 5428 phosphorylation status on control fibroblasts $(n=7)$ and fibroblasts of patients with CS $(n=6)$. (J) Protein content of AMPK $\alpha 1+\alpha 2$, T172_phospho-AMPK, LKB1, and S428_P-LKB1 in heart samples from Costello (HRAs p.G12S) or WT (HRAS) mice. (K) Protein levels of ATC7 and CLPX determined by mass spectrometry in cells from patients with CS $(n=3)$ as compared with control cells $(n=3)$. ( $(\mathrm{L}$ and $\mathbf{M})$ LC3B-I and LC3B-II levels were determined in skin fibroblasts from controls or patients in presence or absence of $300 \mathrm{nM}$ of bafilomycin A1 $(n=3)$. (N) LC3 activation level was expressed as (LC3-II/ [LC3I+LC3II]). (0) Relative normalized expression of TFAM mRNA in cells from patients with CS $(n=3)$. Data are expressed as the mean \pm SEM. One-way ANOVA with Dunnett's correction for multiple testing was used to compare the 3 groups of cells expressing HRAS p.G12A or HRAS p.G12S with the empty plasmid control, while a $t$ test was used to compare the 2 groups of cells obtained from patients with CS and controls or the 2 groups of mice (Costello or WT). ${ }^{*} P<0.05,{ }^{* *} P<0.01,{ }^{* *} P<0.001,{ }^{* * *} P<0.0001$.

with $500 \mu \mathrm{M}$ BZ rescued mitochondrial proteome homeostasis and organelle function in vitro (Figure 7, C-F).

Thus, we performed preclinical treatment of the CS mouse model with BZ $0.05 \%$ (Figure $7 \mathrm{G}$ ). After 12 weeks of treatment with $\mathrm{BZ} 0.05 \%$ in the diet, the enzymatic activity of respiratory chain complex I, complex IV, and citrate synthase was increased (Figure 7G). Moreover, the echocardiography analysis of the treated CS mice revealed that the left ventricle volume was specifically reduced by this treatment (Figure $7, \mathrm{H}$ and I). No significant change was observed in the total mass of the heart (Figure 7J). A substantial reduction of the heart-beating rate was also obtained by the treatment, without modification of the systolic arterial pressure (Figure 7, K and L). Altogether, these findings suggest that the $\mathrm{BZ} 0.05 \%$ treatment prevented development of the left ventricle hypertrophy in the CS mouse model and normalized the heart-beating rate. Still, more specific cardiac functional assessments for reversal/prevention of HCM in CS will need to be performed to prove the therapeutic possibility of using BZ for treating the cardiac disease in CS.

CS is a developmental disorder, so we tested the preclinical strategy of mitochondrial stimulation at an early stage of the disease. To this aim, we generated a GFP-HRASV12 zebrafish model of CS (Figure 8), as previously described (45). Embryos were observed daily from 2 days post fertilization (dpf) to $5 \mathrm{dpf}$ in order to analyze the effect of HRASV12 overexpression on physiological development (Figure 8, A-G). At 2 dpf, 22\% of the HRAS p.G12V embryos died, and this rate increased to reach $60 \%$ mortality at $5 \mathrm{dpf}$ (Figure $8 \mathrm{H}$ ). At $5 \mathrm{dpf}$, among embryos that were alive, $60 \%$ presented developmental defects similar to those observed in humans and mice (Figure 8, G and I) as previously described (45). We observed a cardiac phenotype characterized by heart hypertrophy (12\%) and cardia and pericardia edema (12\%) associated with poorly developed heart and reduced blood flow (12\%). Additional phenotypes were observed, including brain hemorrhage $(6 \%)$ and vascularization defect leading to edema in the duct of Cuvier or malformation of the aorta or the vein in the tail region around the urogenital opening (12\%). The interindividual variability observed within the CS zebrafish population could be explained by the genetic position effect of the HRAS p.G12V transgene, as controlled by the regulatory environment of the genomic integration sites. In order to analyze the effect of mitochondrial bioenergetics and organelle proteostasis modulators on the potential rescue of the observed HRAS p.G12V embryo developmental phenotype, BZ and/or urolithin A (UA) were added to the zebrafish medium (Figure 8, C-F). Animal phenotyping revealed that the $\mathrm{BZ}$ and UA treatments increased the survival rate up to $70 \%$ and $65 \%$ (Figure $8 \mathrm{H}$ ), respectively, and decreased the number of defective embryos among alive embryos (45\% and $43 \%$, respectively) as compared with the vehicle-treated (DMSO) HRAS p.G12V embryos (60\% of defective embryos; Figure 8, F and I). The combination of $10 \mu \mathrm{M} \mathrm{BZ}$ and $5 \mu \mathrm{M}$ UA increased substantially the treatment efficiency by improving embryo survival up to $+30 \%$ (Figure $8 \mathrm{H}$ ) and reduced to a large extent (3-fold) the number of animals presenting genetic developmental defects (Figure $8, \mathrm{~F}$ and $\mathrm{G}$ ), as compared with vehicle-treated embryos. Measurement of HRASV12 plasmid expression by analysis of the level of GFP fluorescence emission confirmed the stable expression of the transgene during the experiments and the absence of effect of the different treatments on the HRASV12 plasmid expression (Supplemental Figure 14A). Finally, molecular investigation of the CS zebrafish model using Western blot performed on whole embryos revealed a 2-fold reduced expression of the mitochondrial marker TOM20 (Figure 8J). The combination treatment composed of $10 \mu \mathrm{M} \mathrm{BZ}$ and $5 \mu \mathrm{M}$ UA restored the expression level of TOM20 in the whole embryo and corrected the defective phenotype (Figure 8, F and I, and Supplemental Figure 14B). A proteomic study performed on whole zebrafish animals treated with $10 \mu \mathrm{M} \mathrm{BZ}$ and $5 \mu \mathrm{M}$ UA (Supplemental Figures 15 and 16) revealed a mode of action composed of at least 3 molecular mechanisms. (a) Bioenergetic stimulation, consisting of a strong stimulation of fatty acid oxidation systems (very-long-chain 3-oxoacyl-CoA reductase-A, carnitine O-palmitoyltransferase, electron transfer flavoprotein subunit $\beta$, etc.), suggested the occurrence of an "oxidative shift." Accordingly, the LXR/RXR pathway that controls fatty acid metabolism was activated by the treatment (Supplemental Figure 16B). (b) Proteostasis modulation, consisting of stimulation of mitochondrial proteostasis machinery, included upregulation of Hsp70, CLPP, YME1-like 1b, COX7A2, ubiquinol-cytochrome $c$ reductase complex assembly factor 1 , and DNAJ (Hsp40 cochaperone) as well as selected proteasome components. The 2 protease CLPP and YME1L1 have a particular interest in our study, as their genetic inhibition induces cardiomyopathy $(23,24)$. (c) Activation of the acute-phase response signaling pathway shows a systemic response aimed at the restoration of tissue homeostasis. In particular, the level of angiotensinogen, the sole precursor of all angiotensin peptides, was increased by a factor of $\log _{2}$ fold $=4$ 
A

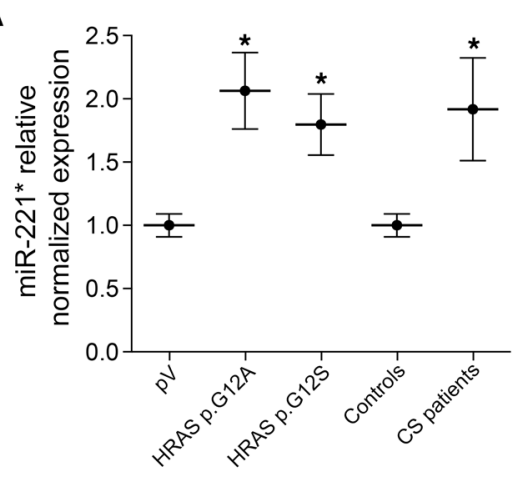

D

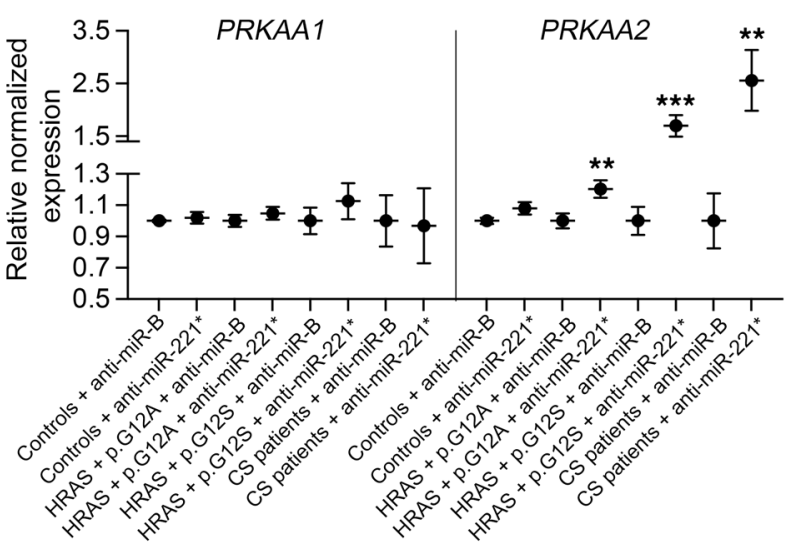

$\mathbf{F}$
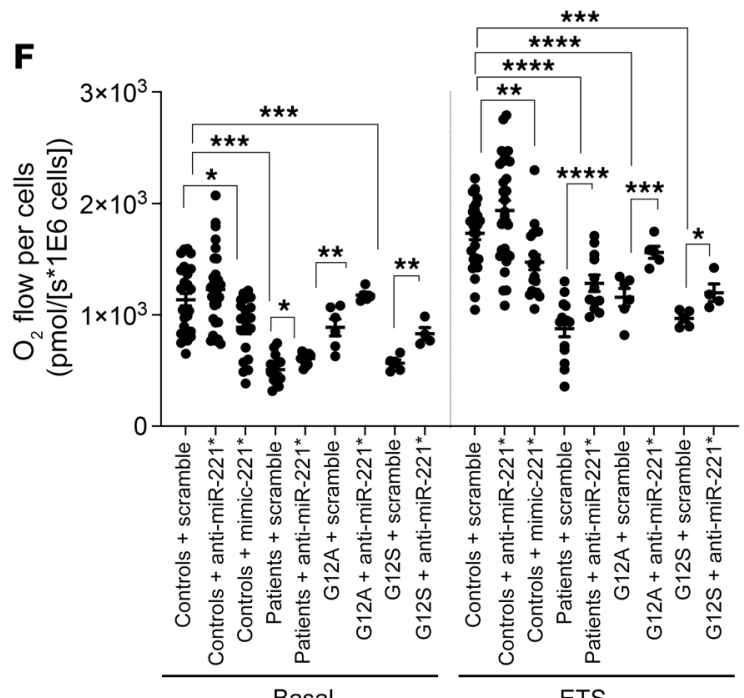

H

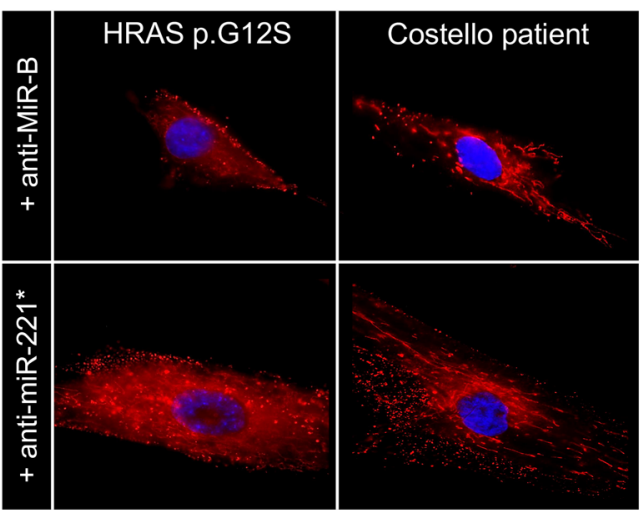

B

HRAS p.G12A

HRAS p.G12S

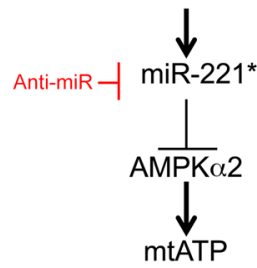

E

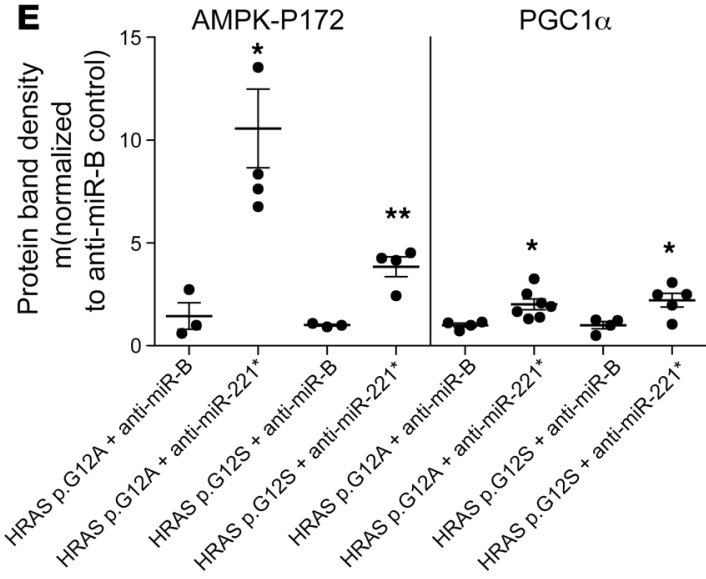

C

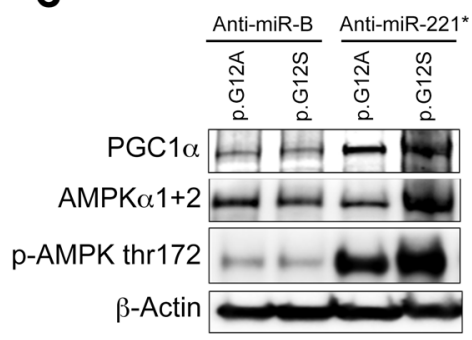


Figure 5. miR-221-5p inhibits AMPKa2 expression in an HRAS-dependent manner. (A) miR-221-5p (miR-221*) expression in skin fibroblasts from patients with CS and related transgenic cell models $(n=3)$. (B) Description of the HRAS/miR-221*/AMPK pathway. (C and D) Rescue of AMPK $\alpha 2$ expression by anti-miR-221-5p at the protein and the mRNA levels, respectively $(n=3)$. (E) Rescue of AMPK $\alpha 2$ expression, T172_P-AMPK/total AMPK ratio, and PGC1 $\alpha$ expression by the anti-miR-221-5p ( $n \geq 3)$. (F) Rescue of mitochondrial respiration by anti-miR-221-5p and its inhibition by a miR-221-5p mimic ( $n \geq 3$ ). (G) Rescue of mitochondrial steady-state ATP content by the anti-miR-221-5p ( $n \geq 3)$. ( $\mathbf{H}$ and $\mathbf{I}$ ) Rescue of mitochondrial particles number by anti-miR-221-5p $(n=3)$. Original magnification, $\times 1000$. Two-way ANOVA with Dunnett's correction for multiple testing was used to compare the 3 groups of cells expressing HRAS p.G12A, HRAS p.G12S, or the empty plasmid control $(\mathrm{pV})$ treated with the anti-miR scramble or the anti-miR-221-5p. Unpaired $t$ test was used to compare the 2 groups of cells obtained from patients with CS and controls. Data are expressed as the mean \pm SEM. ${ }^{*} P<0.05,{ }^{* *} P<0.01,{ }^{* * *} P<0.001$, ${ }^{* * * *} P<0.0001$.

by the treatment. Angiotensin I was also increased by $\log _{2}$ fold $=$ 2.55. Angiotensin I has vasodilator, antidiuretic, antithrombotic, and cardioprotective effects. Finally, the acute-phase response signaling stimulated the expression of members of the complement factors system, also involved in proteostasis. These findings suggest that the strategy of combined mitochondrial bioenergetics and proteostasis stimulation should be further investigated in CS.

\section{Discussion}

Here, we performed a comprehensive analysis of the molecular determinants of CS using untargeted proteomics, bioenergetics, and molecular biology methods on a collection of biological models: tissues of a HRAS p.G12S CS mouse model, skin fibroblasts from patients with CS, CS hiPSC-CMs, human skin fibroblasts transduced with mutant forms of HRAS p.G12S and HRAS p.G12A, and a HRAS p.G12V zebrafish model of CS. Confrontation of these models allowed us to better understand the molecular impact of mutant HRAS in different contexts and to reveal tissue-specific changes. The original CS mouse model used in our study was generated by a knockin of a mutant HRAS p.G12S gene in C57BL/6 animals and developed HCM. All the CS mouse and zebrafish models reported in the literature develop HCM (7, $10,16)$, and this clinical feature can be prognostic of worsening disease for patients.

Using in vitro and in vivo CS biological models, we discovered that germline or somatic HRAS p.G12S and p.G12A mutations alter mitochondrial proteome homeostasis and OXPHOS in a tissue-specific manner. For instance, CS mice exhibited downregulation of OXPHOS components in the heart and the liver, while the brain and, to a lesser extent, the skeletal muscle, showed accumulation of ETC proteins. The CS ectopic cell models (HRAS p.G12A and p.G12S) also exhibited a profile of OXPHOS protein accumulation associated with defective mitochondrial function. We further validated these findings in cardiomyocytes generated from the differentiation of hiPSCs from patients with CS. Taken together, our observations raised the hypothesis of defective mitochondrial homeostasis in CS.

Thus, we investigated the potential implication of AMPK in CS molecular pathophysiology and mitochondrial dysfunction, based on the pathway analysis performed on the differential pro- teome determined in the different CS mouse tissues and cellular models. Biochemical studies also showed that AMPK controls mitochondrial protein biogenesis and degradation $(46,47)$. AMPK dysfunction and mitochondrial respiratory chain defects are also frequent causes of HCM $(17-22,48)$. Our results revealed a strong inhibition of AMPK expression level (mRNA and protein) in heart samples from CS animals, in skin fibroblasts from patients with $\mathrm{CS}$, in cell models overexpressing mutant forms of HRAS, and in CS hiPSC-CMs. These consistent findings support the hypothesis of defective AMPK signaling in CS. The interplay between RAS/ MAPK and AMPK signaling was investigated in previous studies (49) and revealed the inhibition of the latter by the former, by at least 3 mechanisms: (a) LKB1 inhibition, (b) inhibition of AMPK catalytic activity, and (c) inhibition of AMPK by KSR2. However, little is known about the interplay between the HRAS/MAPK and the LKB1/AMPK pathway in the context of RASopathies. Our results revealed the inhibition of PRKAA2 mRNA expression in skin fibroblasts from patients with CS, CS mouse hearts, and hiPSC-CMs. The $\alpha 2$ subunit of AMPK is essential for AMPK signaling because its phosphorylation on Thr172 activates the kinase. Moreover, a reduction of LKB1 protein content was also found in skin fibroblasts from patients with CS and CS mouse hearts, suggesting a dual inhibition of the LKB1/AMPK signaling pathway in CS. The observed reduction of LKB1_Ser428 phosphorylation level in fibroblasts from patients with CS and in CS mouse hearts suggests an even more upstream regulatory mechanism, whereby altered activity of kinases upstream of LKB1 might contribute to the decreased AMPK (thr172) phosphorylation observed. Moreover, LKB1 has numerous phosphorylation sites and the sole change in the phosphorylation level of Ser428 is not sufficient to evaluate LKB1 activity. Alterations in LKB1 interactions with other proteins or changes in its subcellular distribution could also occur.

We also discovered that the inhibition of PRKAA2 mRNA expression by HRAS p.G12S was mediated by micro-RNA-211-5p $\left(\operatorname{miR}-221^{*}\right)$. Thus, we searched for a possible effect of miR-221* on PRKAA2 expression in the context of CS. We observed that inhibition of miR-221* rescued cellular energy homeostasis, suggesting that anti-miR-221 strategies could be considered in future studies. However, we were not able to detect the endogenous level of miR-221* in the hearts of WT or CS mice. Technically, the total pool of microRNAs was extracted in sufficient quantity and quality from the CS mouse heart, but amplification of miR-221* with a validated commercially available primer (Qiagen miScript Primer Assay) could not reach the detection threshold. One explanation for this finding could be that the microRNA miR-221-3p is differentially regulated in mouse and human cardiac pathology (50). Moreover, genetic studies investigating the role of miR-221 in the heart made use of transgenic mice overexpressing miR-221, not taking into account endogenous miRNA levels, as we tried to do (51-53). Finally, comparative analysis of microRNAs between humans and mice showed a significant level of divergence in the heart (54). For these reasons, we restrained our explanation of the molecular mechanism responsible for the mitochondrial defective phenotype of CS to the HRAS-mediated inhibition of LKB1/ AMPK signaling. However, additional studies will be required to fully understand how altered LKB1 and AMPK signaling and miR-221* increased expression could be responsible for the broad 

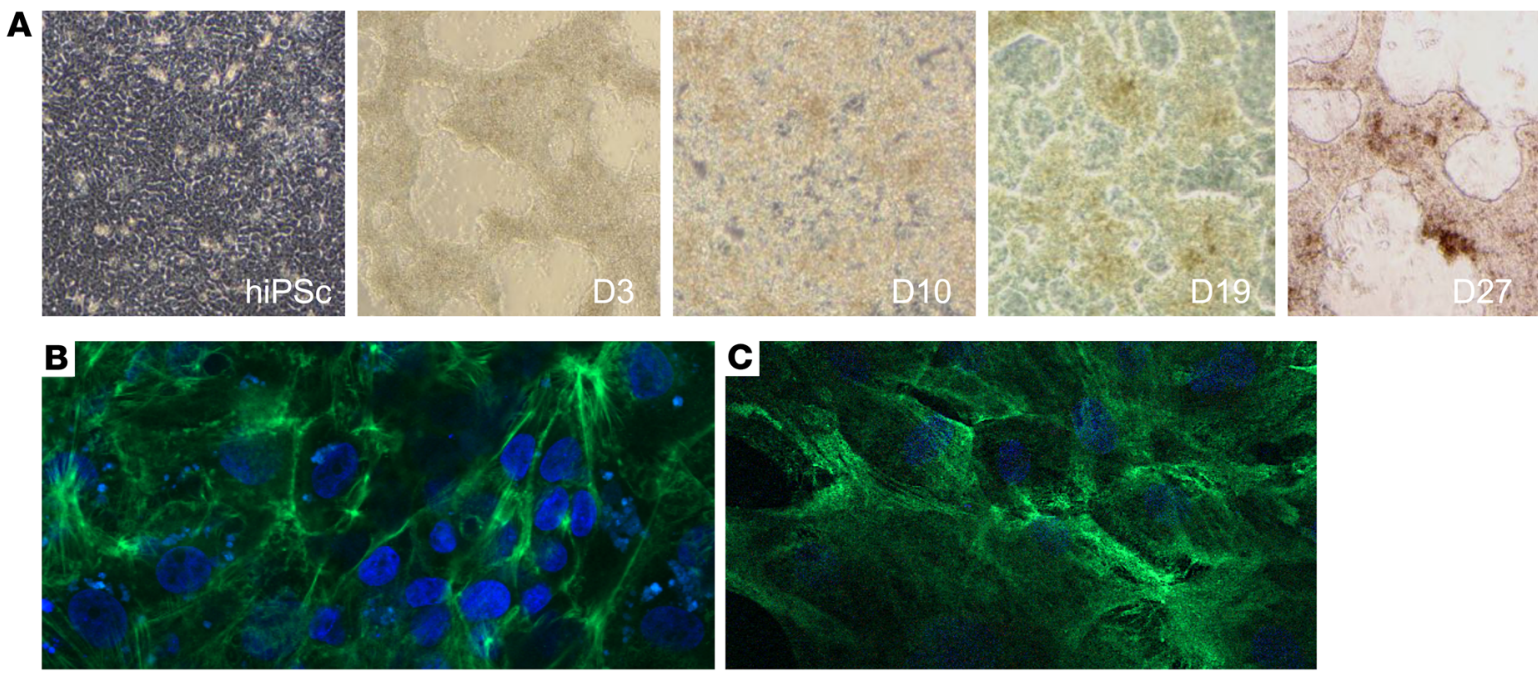

D

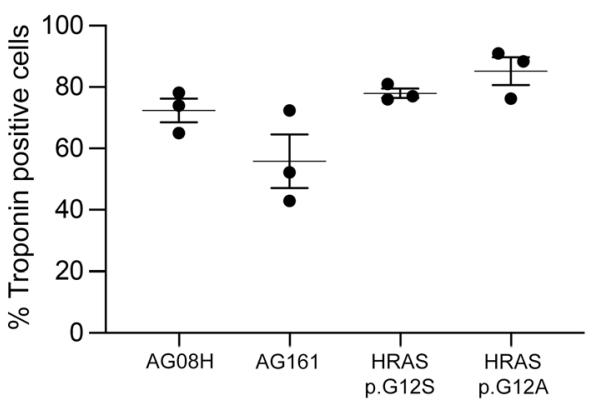

G

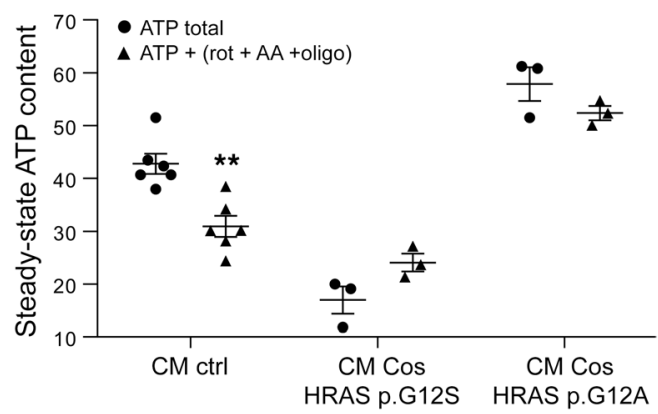

J

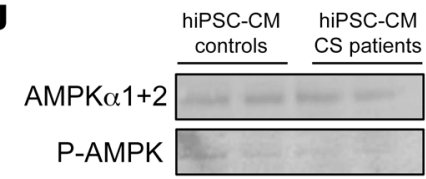

P-LKB1 (S428)

GAPDH
E

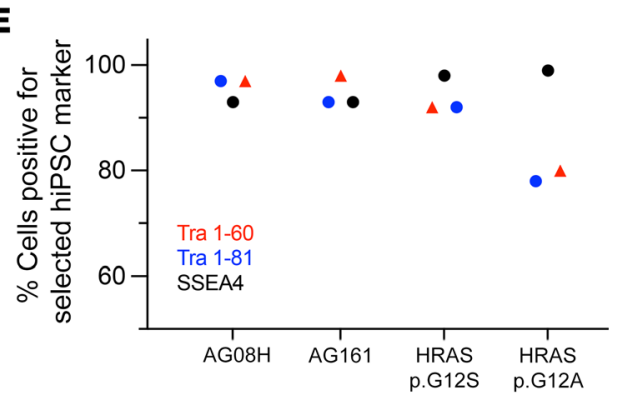

I

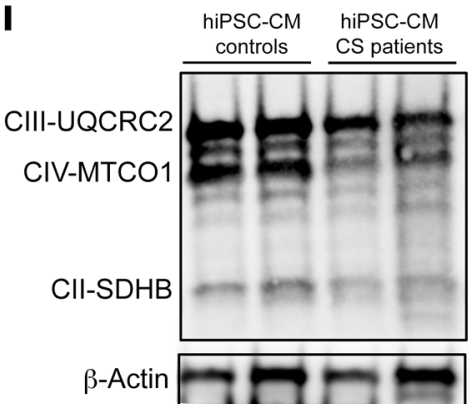

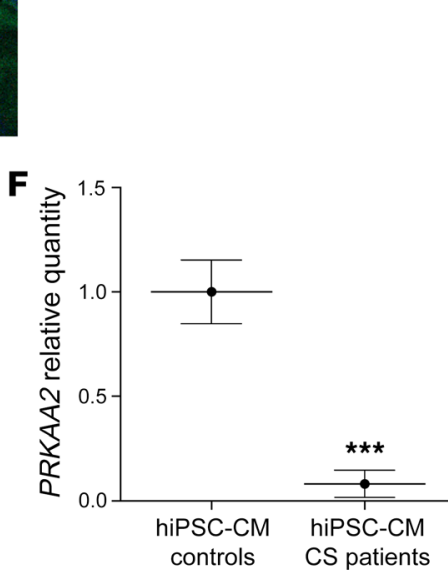

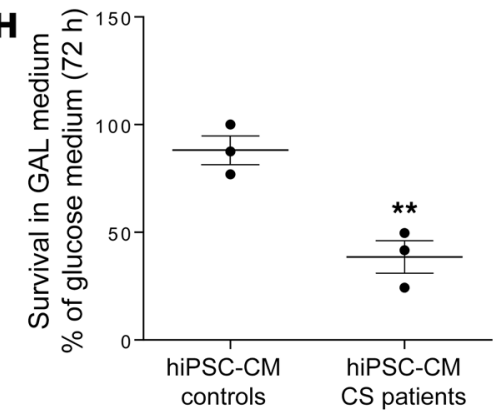

\section{(1)}



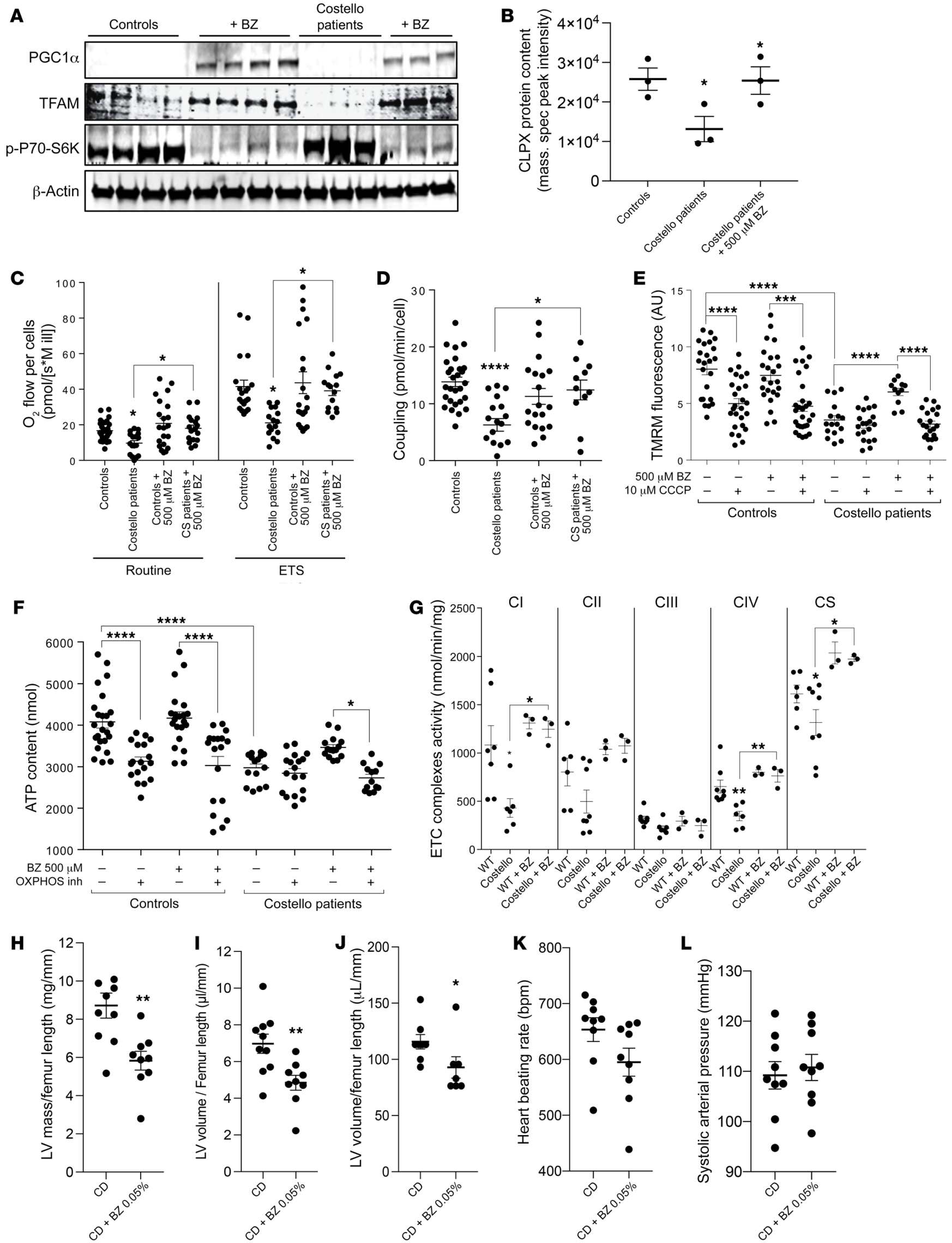
Figure 7. Bezafibrate rescues mitochondrial bioenergetics and prevents left ventricle cardiac hypertrophy in Costello syndrome. (A) Increased expression of PGC1 $\alpha$, TFAM, and p-P70S6K by bezafibrate $(500 \mu \mathrm{M} ; 48$ hours) treatment in skin fibroblasts from patients with CS. (B) CLPX protein content determined by mass spectrometry of cells from controls, cells from patients with CS, and bezafibrate-treated cells from patients with CS. Quantification was expressed as the normalized peak intensity $(n=3)$. (C-F) Defective mitochondrial respiration, OXPHOS coupling, mitochondrial transmembrane electric potential, and mitochondrial ATP levels were rescued by bezafibrate ( $500 \mu \mathrm{M} ; 48$ hours) in skin fibroblasts from patients with CS $(n=3)$. (G) Stimulation of respiratory chain complex enzymatic activity in the hearts of CS mice treated with bezafibrate $0.05 \%$ in the diet (for 12 weeks). Effect of the bezafibrate $0.05 \%$ in chow diet (CD + BZ $0.05 \%$ ) on (H) left ventricle (LV) mass, (I) left ventricle volume, (J) heart mass, (K) heart beating rate, and (L) systolic arterial pressure after 12 weeks of treatment in CS mouse model $(n=9)$ as compared with untreated CS mice fed with chow diet (CD) $(n=9)$. One-way ANOVA with Dunnett's correction for multiple testing was used to compare the 4 groups of cells (controls treated or untreated with bezafibrate and CS mice treated or untreated with bezafibrate). Unpaired $t$ test was used to compare the 2 groups of mice (WT or Costello). ${ }^{*} P<0.05,{ }^{* *} P<0.01,{ }^{* * *} P<0.001,{ }^{* * *} P<0.0001$.

spectrum of clinical symptoms of CS. miR-221* targets downregulated in fibroblasts from patients with CS could also play a role in CS pathophysiology, but this mechanism remains to be demonstrated in CS mouse heart. miR-221* targets are involved in cell migration, proliferation, and adhesion, key processes required for neural crest cell patterning in craniofacial development. Future studies could consider the role of miR-221* in the determination of the craniofacial features common in CS.

A major impact of the LKB1/AMPK inhibition observed in the different CS models investigated in our study is the alteration of mitochondrial proteostasis, albeit with tissue-specific differences. The causality link between AMPK signaling and the control of mitochondrial proteostasis was established in previous studies $(46,55,56)$. Our analysis of the molecular mechanisms underlying the alteration of mitochondrial proteome and bioenergetics in skin fibroblasts from patients with CS and CS mouse hearts showed the inhibition of key executors and regulators of mitochondrial turnover to be AMPK, SQSTM1/P62, and CRIF1. These proteins play a role in mitophagy, and biochemical studies have shown that AMPK $\alpha 2$ coordinates this process and thereby protects against heart failure $(57,58)$, in complete agreement with our findings. Mechanistically, we observed a tissue-specific alteration in the machinery responsible for mitochondrial proteostasis, a very complex and intricate network of quality and quantity control mechanisms (59) recently considered as a therapeutic target in myocardial infarction (60). In the CS cell models, we further observed the altered expression of the autophagy regulators (ATG7) and targeted mitochondrial degradation (CLPX) but also that of central mediators of mitochondrial biogenesis (such as TFAM), suggesting defective mitochondrial proteostasis and biogenesis in cells from patients with CS. Our hypothesis was confirmed by the observation of reduced autophagic flux in cells from patients with CS, accumulation of mitochondrial proteins, and defective organelle bioenergetics. The mitochondrial protein CLPX reduced in cells from patients with CS is the ATP-dependent Clp protease ATP-binding subunit. The ClpP-ClpX (CLpXP) complex is a central regulator of mitochondrial proteostasis and bioenergetics $(27,28,61,62)$. Our observation of CLPX downregulation by mutant HRAS in CS could suggest further study of the link between the RAS/MAPK pathway and CLpXP.

The variable alteration of the mitochondrial proteostasis machinery and of the mitochondrial proteome itself, observed between the different tissues of the CS mouse, raised the question of the tissue-specific impact of HRAS germline mutations. There are still questions regarding the degree of functional tissue specificity exhibited by mutant HRAS in various cell types and tissues, and previous studies have shown that germline HRAS mutation alters the development of the different tissues to variable extents (63). For instance, the developmental phenotypes of patients with CS and CS animal models indicate a strong tissue-specific effect of the germline HRAS mutations on the craniofacial development, heart structure and function, skin, and predisposition to specific cancers. This tissue-specific impact of HRAS germline mutations was partly explained by the specific role of the different RAS isoforms in tissues of variable embryological origins (64, 65). Differences in the interactions with regulators and effectors of the RAS signaling pathway also participate to the tissue-specific functions of the different RAS isoforms. The LKB1/AMPK signaling involved in CS molecular pathophysiology is also a tissuespecific pathway $(58,66,67)$. In particular, the control of mitochondrial biogenesis and degradation by AMPK is variable between tissues. We measured a reduced TFAM content in HRAS mutant cell models and in the CS mouse heart. However, the reduction in PGC1 $\alpha$ was only detected in the mutant HRAS cell models. This result could be explained by the fact that PGC1 $\alpha$ is typically regulated by deacetylation in muscle, rather than through changes in expression (68). Another tissue-specific concern of our study was the use of skin fibroblasts that present with very different bioenergetic features as compared with cardiomyocytes. Previous studies in the field of mitochondrial diseases have shown that skin fibroblasts do not recapitulate the bioenergetic dysfunction found in the skeletal muscle (69). The use of hiPSC-CMs and of CS mouse hearts was instrumental to validating our findings. In particular, we found that most of the ATP produced in CS hiPSC-CMs was obtained from glycolysis, as previously observed in conditions of cardiac disease and OXPHOS dysfunction $(70,71)$. However, in the context of CS, the skin fibroblasts constitute a relevant cell model, as the skin expresses the disease in various forms (72). Conversely, while hiPSC-CMs from patients with CS provide a pertinent model allowing comparison with CS mouse hearts and human hearts, the growth conditions of the cardiomyocytes will directly impact their bioenergetics and may not provide the best environment to study the mechanism underlying OXPHOS alteration. In particular, the medium used for the maintenance of hiPSC-CMs contains a large number of growth factors and high concentrations of energy substrates, which limits the study of the LKB1/AMPK pathway. To conclude, we think that studying the mechanisms in the CS mouse heart may provide a more reliable data set for understanding the pathophysiology of CS cardiac disease; the cell models used in our study, which all revealed a constitutive mitochondrial dysfunction, present specific bias for the mechanistic investigations. Moreover, our discovery of altered LKB1/AMPK signaling 


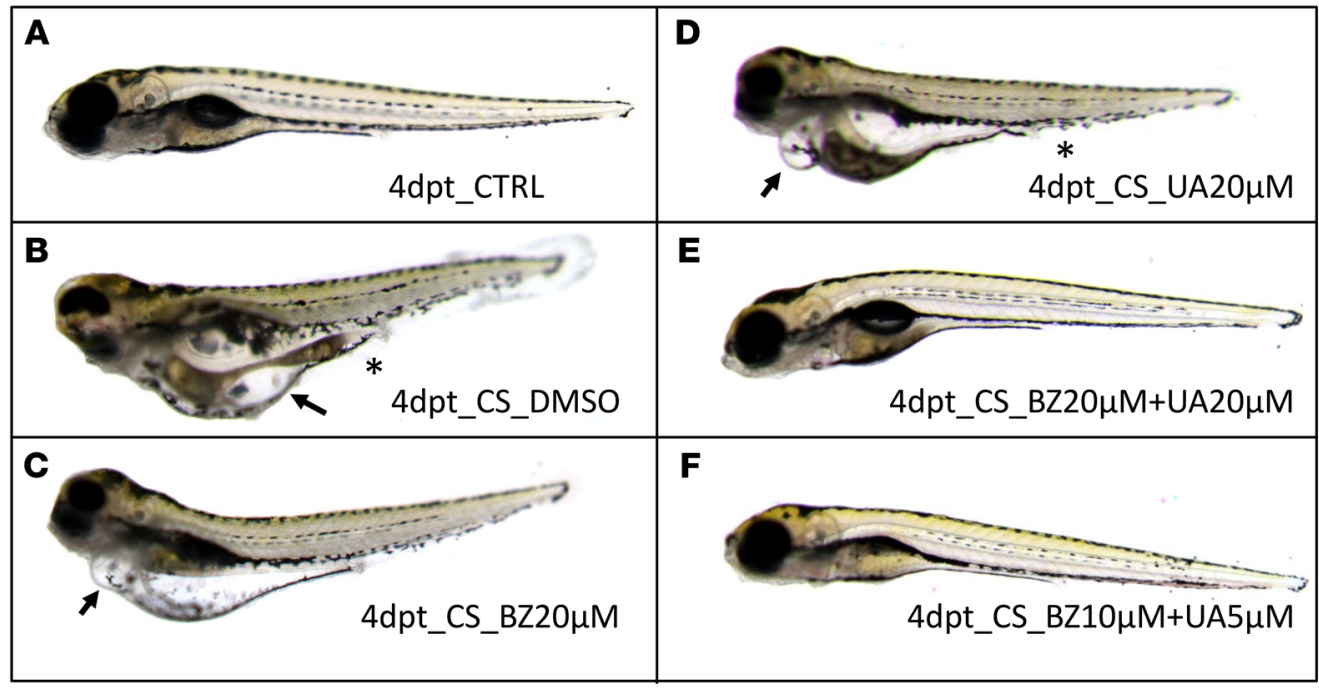

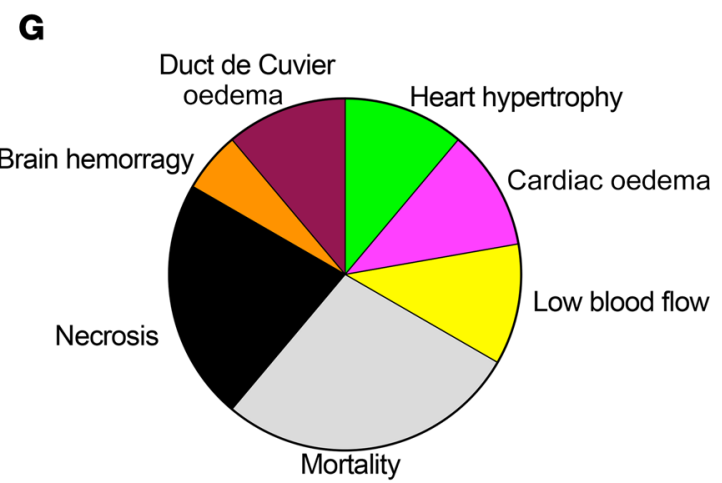

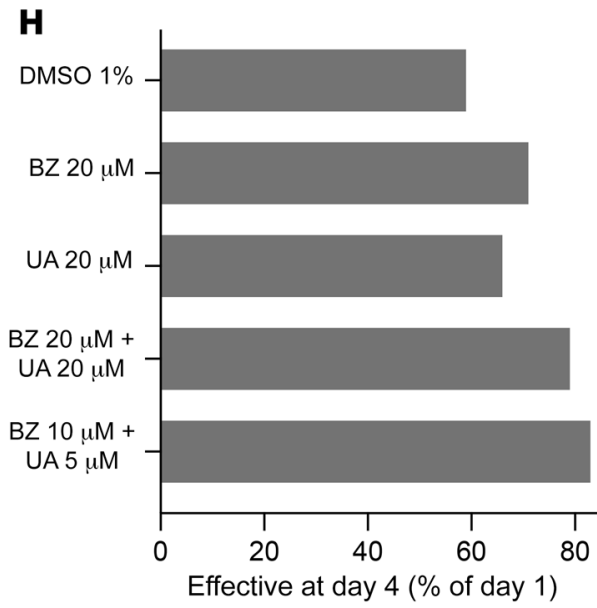

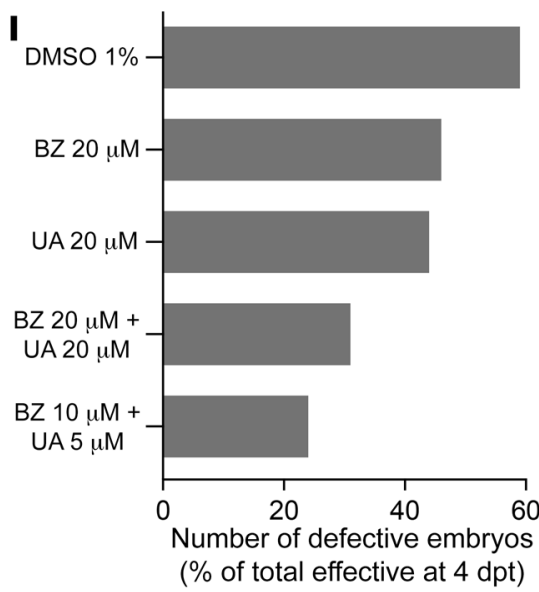

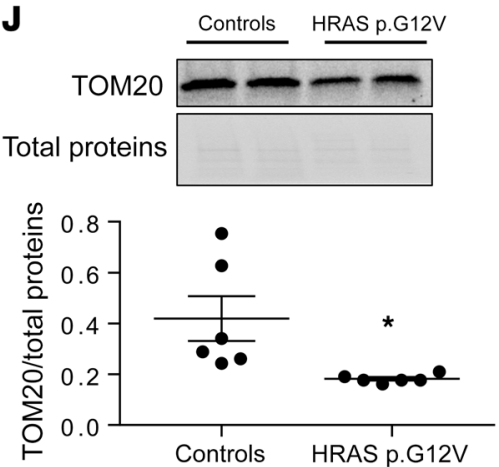

Figure 8. Combination of bezafibrate and urolithin A restores normal phenotype in Costello zebrafish. Phenotype analysis of (A) control zebrafish or (B-H) Costello zebrafish injected with HRASV12 plasmid at $5 \mathrm{dpf}$, after 4 days of treatment with (B) DMSO, (C) bezafibrate, (D) urolithin A, or (E and F) bezafibrate (BZ) and urolithin A (UA) in combination. Costello zebrafish developed edema (B-D, arrows) or hemorrhages and vascularization defects (B and D, asterisks). (F) Treatment with a combination of $10 \mu \mathrm{M} \mathrm{BZ}$ and $5 \mu \mathrm{M}$ UA markedly reduced the percentage of abnormal embryos. (G) Illustration of the phenotypes observed in Costello zebrafish at $5 \mathrm{dpf}$ after expression of HRASV12 plasmid. (H) Expression level of the mitochondrial protein TOM20 in control or HRAS p.G12V embryos. TOM20 protein content determined by Western blot was normalized to the total protein content. (I) Survival rate of Costello zebrafish after 4 days of treatment with DMSO, bezafibrate, or UA alone or in combination. Data were normalized to day 1 of treatment. (J) Percentage of defect appearance in Costello zebrafish after 4 days of treatment with DMSO, bezafibrate, or UA alone or in combination. Data are expressed as number of embryos or as the mean value of TOM20 expression. Unpaired t test was used to compare the 2 groups of zebrafish (WT or Costello). ${ }^{*} P<0.05$. 
and defective mitochondrial proteostasis and bioenergetics only explains one facet of the complexity of CS molecular pathophysiology. For instance, Prkaa2-KO mice show no cardiac alteration, and these mice show exacerbated pressure overload-induced left ventricular hypertrophy only under stress conditions (73). Likewise, we found a small activation of AMPK $\alpha 2$ (p-thr172) and a consecutive low inhibition of ACC (p-ser79) in the CS mouse and cell models investigated under routine conditions.

In this study, we discovered a reduction of LKB1 protein content in CS mouse hearts and skin fibroblasts from patients with CS; this is in agreement with results from a previously published work that showed that genetic deletion of $L K B 1$ in the heart triggers hypertrophy and cardiac dysfunction (74). These findings suggest that AMPK inhibition alone cannot induce heart dysfunction and that additional stress, as mediated by the deregulated HRAS/ MAPK pathway, or a challenging bioenergetic environment must occur. A series of transcriptomic targets have been identified in response to AMPK activation. For instance, AMPK was shown to phosphorylate the transcription factor FoxO3 on 6 serine residues to enhance its transcriptional activity (75) toward its target genes. Growing evidence suggests that in the long-term AMPK promotes metabolic reprogramming via effects on gene expression at least partly through regulation of specific transcription factors and transcriptional coactivators (31). In agreement with this view, analysis of CS heart proteome revealed a complex molecular signature of cardiac dilation and damage, apparently independent of AMPK signaling. Finally, previous metabolic investigation in a HRAS p.G12S CS mouse model (10) revealed a decrease in fatty acid oxidation enzyme expression in the liver, in agreement with the inhibition of LKB1/AMPK signaling observed in our study.

Finally, our work demonstrated that BZ could rescue or prevent the defective mitochondrial function in skin fibroblasts from patients with CS and CS mouse hearts. We discovered that the mode of action of BZ in vitro is multiple and involves the stimulation of PGC1 $\alpha$ expression, mTOR inhibition, and activation of LC3II-mediated autophagy. In particular, BZ rescued the level of the mitochondrial proteostasis regulator ClpX, which was downregulated in CS mouse hearts and cell models. We treated the CS mouse with $0.05 \% \mathrm{BZ}$ in the diet based on a study showing cardiac beneficial effects in a mouse model of the mitochondrial disease Barth syndrome (42). Other rodent studies using a higher dose of BZ also showed OXPHOS improvement and beneficial effects in various mouse models with mitochondrial dysfunction (40, 76-81). However, hepatomegaly was observed when using suprapharmacological doses of $\mathrm{BZ}$ in mice, although this effect was not observed in humans (40). BZ is well tolerated in humans, and clinical trials have revealed that this drug can reduce the heart rate and blood pressure in patients with hypertriglyceridemia (82). BZ also improved the condition of patients with hyperlipidemia (83) and fatty oxidation defect (41). Furthermore, a recent trial on 6 patients with a genetic mitochondrial disease revealed an improved cardiac function (84). To further evaluate the potential of mitochondrial bioenergetics and proteostasis modulation in the treatment of CS in the early stage of the disease we tested the efficacy UA alone or in combination with $\mathrm{BZ}$ in a previously described CS zebrafish model (45). The mitochondrial proteostasis modulator UA was recently characterized and showed no toxicity in a human clinical trial (85-87). We discovered that UA in combination with $\mathrm{BZ}$ synergistically increased the survival of CS embryos and significantly reduced the occurrence of developmental defects. The mode of action of this combination of drugs involved the stimulation of fatty acid oxidation, the activation of several mitochondrial proteostasis regulators and executors, and the stimulation of the acute-phase response, which involved regulation of the angiotensin system. Of interest for our study, previous work showed that AMPK regulates the renin-angiotensin system, and the observed alteration of AMPK signaling in CS could also participate in the cardiac disease through this system (88).

Therefore, our study provides preclinical and molecular evidence that stimulation of mitochondrial quantity control and bioenergetics, as obtained with BZ and UA treatment, improved the health of cellular and animal models of CS. A more detailed heart structure and function analysis with additional clinically relevant data will be required before the use of $\mathrm{BZ}$ and $\mathrm{UA}$ in combination for the prevention of cardiac disease in CS is proposed. To conclude, our work supports the view that mitochondrial quality surveillance is a therapeutic target in heart dysfunction in CS.

\section{Methods}

Further information can be found in Supplemental Methods.

Study approval. All patients gave written informed consent to participate in this study, according to the Committee for the Protection of Persons (University of Bordeaux, Bordeaux, France) (IDRCB no. 2015-A00705-44, CPP 2014/44, ClinicalTrials.gov NCT02812511). All the zebrafish studies conducted for this manuscript have been reviewed and approved by the Comité d'éthique Langedoc Roussilon (Université de Montpellier, Montpellier, France) (F341725/210632019061317218694). The mouse preclinical studies were performed in accordance with the European guidelines for animal experimentation at the Institut Clinique de la Souris, Illkirch-Graffenstaden, France. All the mouse studies conducted for this manuscript have been reviewed and approved by the comité d'éthique Com'Eth, Illkirch, France.

\section{Author contributions}

$\mathrm{RR}, \mathrm{CH}, \mathrm{TS}$, and LD participated in the conception and design of the study. LD and $\mathrm{CH}$ (gene and miR expression studies); LD, NDA, and NB (patients with CS and mutant HRAS bioenergetics properties); ED (respiratory chain enzymes activities); GBA, $\mathrm{YH}$, and TS (BZ preclinical study on the CS mouse model); and CA and WB (proteomics) performed the experiments. RR and LD performed bioinformatics (IPA, Qiagen) and statistical (Prism) analyses of the data. VGP produced the lentiviral plasmid for HRAS mutant ectopic expression. PE participated in the bioenergetics analyses. SC and MB provided expertise in proteomics. PES and JBT performed the cardiology investigations on patients with CS. LF and KK performed the zebrafish study at Azelead. FM, $\mathrm{NL}, \mathrm{MD}$, and LB produced the hiPSCs and their differentiation in cardiomyocytes. They also performed Western blotting on these cells. DL performed diagnostics studies, genetic identification, and patient management. DL, NB, and RR obtained the agreement to generate the cell lines from patients with CS used in this study (ClinicalTrials.gov NCT02812511). RR supervised the entire project and wrote the paper along with LD and NDA, with suggestions from all authors. 


\section{Acknowledgments}

We are very grateful to the French Association of Costello Syndrome and Cardiofaciocutaneous Syndrome, which supported this study in two ways. This association informed patients with CS about the need for skin samples for research and funded the generation of the HRAS p.G12S CS mouse model at the Mouse Clinical Institute (http://www.ics-mci.fr/en/) under the supervision of TS and YH. We are indebted to all the families of patients with CS who provided consent to allow us to perform skin biopsies on their children. We thank the children with CS and Cardiofaciocutaneous Syndrome who participated in this study for their efforts and dedication to this project. We are grateful to Marina Mione from the Department of Cellular, Computational and Integrative Biology, University of Trento, for providing the GFPH-RASV12 plasmid used to generate the CS zebrafish model. We also thank Marie-Laure Martin-Négrier from the Department of Anatomopathology of Bordeaux hospital for her help in the histo-enzymologic study of CS mouse muscle. We thank Fatima Djouadi and Jean Bastin for discussions on BZ's mode of action. We acknowledge funding by the French National Research Agency (ANR) through the COSMIT program (pathophysiology of CS, ANR-15-CE17-0003). Our project was also supported by funding from the Institut National de la Santé et de la Recherche Médicale (INSERM) and the University of Bordeaux. Development of the drug combination BZ-UA is supported by SATT Nouvelle-Aquitaine "AST." We are grateful to our funders for providing support these last years.

Address Correspondence: Rodrigue Rossignol, INSERM U1211, MRGM Laboratory, Bordeaux University Hospital (CHU), place amélie Raba-Léon 33000 Bordeaux, France. Phone: 33.0.5.57.82.10.54; Email rodrigue.rossignol@u-bordeaux.fr.
1. Costello J. A new syndrome. NZ Med J. 1971;74:397.

2. Lin AE, et al. Clinical, pathological, and molecular analyses of cardiovascular abnormalities in Costello syndrome: a Ras/MAPK pathway syndrome. Am JMed Genet A. 2011;155(3):486-507.

3. Siwik ES, et al. Cardiac disease in Costello syndrome. Pediatrics. 1998;101(4 pt 1):706-709.

4. Leoni C, et al. Musculo-skeletal phenotype of Costello syndrome and cardio-facio-cutaneous syndrome: insights on the functional assessment status. Orphanet J Rare Dis. 2021;16(1):43.

5. Wei BR, et al. Capacity for resolution of Ras-MAPK-initiated early pathogenic myocardial hypertrophy modeled in mice. Comp Med. 2011;61(2):109-118.

6. Hunter JJ, et al. Ventricular expression of a MLC$2 \mathrm{v}$-ras fusion gene induces cardiac hypertrophy and selective diastolic dysfunction in transgenic mice. J Biol Chem. 1995;270(39):23173-23178.

7. Schuhmacher AJ, et al. A mouse model for Costello syndrome reveals an Ang II-mediated hypertensive condition. J Clin Invest. 2008;118(6):2169-2179.

8. Gottshall KR, et al. Ras-dependent pathways induce obstructive hypertrophy in echo-selected transgenic mice. Proc Natl Acad Sci U S A. 1997;94(9):4710-4715.

9. Sana ME, et al. A novel HRAS mutation independently contributes to left ventricular hypertrophy in a family with a known MYH7 mutation. PLoS One. 2016;11(12):e0168501.

10. Oba D, et al. Mice with an oncogenic HRAS Mutation are resistant to high-fat diet-induced obesity and exhibit impaired hepatic energy homeostasis. EBioMedicine. 2018;27:138-150.

11. Ormerod JOM, et al. Myocardial energy depletion and dynamic systolic dysfunction in hypertrophic cardiomyopathy. Nat Rev Cardiol. 2016;13(11):677-687.

12. Kloner RA, et al. New and revisited approaches to preserving the reperfused myocardium. Nat Rev Cardiol. 2017;14(11):679-693.

13. McManus MJ, et al. Mitochondrial DNA variation dictates expressivity and progression of nuclear DNA mutations causing cardiomyopathy. Cell Metab. 2019;29(1):78-90.
14. Murphy E, et al. Mitochondrial function, biology, and role in disease. Circ Res. 2016;118(12):1960-1991.

15. Kleefstra T, et al. Mitochondrial dysfunction and organic aciduria in five patients carrying mutations in the Ras-MAPK pathway. Eur J Hum Genet. 2011;19(2):138-144.

16. Dard L, et al. RAS signalling in energy metabolism and rare human diseases. Biochim Biophys Acta Bioenerg. 2018;1859(9):845-867.

17. $\mathrm{Hu} \mathrm{X}$, et al. AMP activated protein kinase-2 regulates expression of estrogen-related receptor-, a metabolic transcription factor related to heart failure development. Hypertension. 2011;58(4):696-703.

18. Arany Z, et al. Transcriptional coactivator PGC- $1 \alpha$ controls the energy state and contractile function of cardiac muscle. Cell Metab. 2005;1(4):259-271.

19. Kärkkäinen O, et al. Heart specific PGC-1 $\alpha$ deletion identifies metabolome of cardiac restricted metabolic heart failure. Cardiovasc Res. 2019;115(1):107-118.

20. Erkens R, et al. Left ventricular diastolic dysfunction in Nrf2 knock out mice is associated with cardiac hypertrophy, decreased expression of SERCA2a, and preserved endothelial function. Free Radic Biol Med. 2015;89:906-917.

21. Huss JM, et al. The nuclear receptor ERRalpha is required for the bioenergetic and functional adaptation to cardiac pressure overload. Cell Metab. 2007;6(1):25-37.

22. Luptak I, et al. Decreased contractile and metabolic reserve in peroxisome proliferator-activated receptor-alpha-null hearts can be rescued by increasing glucose transport and utilization. Circulation. 2005;112(15):2339-2346.

23. Seiferling D, et al. Loss of CLPP alleviates mitochondrial cardiomyopathy without affecting the mammalian UPRmt. EMBO Rep. 2016;17(7):953-964.

24. Wai T, et al. Imbalanced OPA1 processing and mitochondrial fragmentation cause heart failure in mice. Science. 2015;350(6265):aad0116.

25. Margonato D, et al. Tricuspid regurgitation in left ventricular systolic dysfunction: marker or target? Front Cardiovasc Med. 2021;8:649.
26. Sara R, et al. Altered cardiac energetics and mitochondrial dysfunction in hypertrophic cardiomyopathy. Circulation. 2021;23(144):1714-1731.

27. Cole A, et al. Inhibition of the mitochondrial protease $\mathrm{ClpP}$ as a therapeutic strategy for human acute myeloid leukemia. Cancer Cell. 2015;27(6):864-876.

28. Deepa SS, et al. Down-regulation of the mitochondrial matrix peptidase $\mathrm{ClpP}$ in muscle cells causes mitochondrial dysfunction and decreases cell proliferation. Free Radic Biol Med. 2016;91:281-292.

29. Nahapetyan $\mathrm{H}$, et al. Altered mitochondrial quality control in Atg7-deficient VSMCs promotes enhanced apoptosis and is linked to unstable atherosclerotic plaque phenotype. Cell Death Dis. 2019;10(2):119.

30. Guo JY, White E. Autophagy is required for mitochondrial function, lipid metabolism, growth, and fate of KRAS(G12D)-driven lung tumors. Autophagy. 2013;9(10):1636-1638.

31. Collodet C, et al. AMPK promotes induction of the tumor suppressor FLCN through activation of TFEB independently of mTOR. FASEB J. 2019;33(11):12374-12391.

32. Léveillé $\mathrm{M}$, et al. PGC-1 $\alpha$ isoforms coordinate to balance hepatic metabolism and apoptosis in inflammatory environments. Mol Metab. 2020;34:72-84.

33. Stinson S, et al. TRPS1 Targeting by miR-221/222 promotes the epithelial-to-mesenchymal transition in breast cancer. Sci Signal. 2011;4(177):ra41.

34. Liu CW, et al. Resveratrol attenuates ICAM-1 expression and monocyte adhesiveness to TNF- $\alpha$-treated endothelial cells: evidence for an anti-inflammatory cascade mediated by the miR221/222/AMPK/p38/NF-кB pathway. Sci Rep. 2017;7(1):44689.

35. Yang $\mathrm{Y}$, et al. miR-221-3p involves in the invasion of trophoblast and angiogenesis through AMPK pathway and leads to unexplained recurrent spontaneous abortion. Int J Clin Exp Pathol. 2016;9(11):11092-11098.

36. Tsunoda T, et al. Oncogenic KRAS regulates miR-200c and miR-221/222 in a 3D-specific manner in colorectal cancer cells. Anticancer Res. 2011;31(7):2453-2459. 
37. Kakimoto Y, et al. Overexpression of miR-221 in sudden death with cardiac hypertrophy patients. Heliyon. 2018;4(6):e00639.

38. Rossignol R, et al. Energy substrate modulates mitochondrial structure and oxidative capacity in cancer cells. Cancer Res. 2004;64(3):985-993.

39. Huang Y, et al. The PPAR pan-agonist bezafibrate ameliorates cardiomyopathy in a mouse model of Barth syndrome. Orphanet J Rare Dis. 2017;12(1):49.

40. Djouadi F, Bastin J. Species differences in the effects of bezafibrate as a potential treatment of mitochondrial disorders. Cell Metab. 2011;14(6):715-716.

41. Bonnefont JP, et al. Bezafibrate for an inborn mitochondrial beta-oxidation defect. $N$ Engl J Med. 2009;360(8):838-840.

42. Schafer $\mathrm{C}$, et al. The effects of PPAR stimulation on cardiac metabolic pathways in barth syndrome mice. Front Pharmacol. 2018;9:318.

43. Buzzai M, et al. The glucose dependence of Akt-transformed cells can be reversed by pharmacologic activation of fatty acid beta-oxidation. Oncogene. 2005;24(26):4165-4173.

44. Waskowicz LR, et al. Bezafibrate induces autophagy and improves hepatic lipid metabolism in glycogen storage disease type Ia. Hum Mol Genet. 2019;28(1):143-154.

45. Santoriello C, et al. Expression of H-RASV12 in a zebrafish model of Costello syndrome causes cellular senescence in adult proliferating cells. Dis Model Mech. 2009;2(1-2):56-67.

46. Mihaylova MM, Shaw RJ. The AMPK signalling pathway coordinates cell growth, autophagy and metabolism. Nat Cell Biol. 2011;13(9):1016-1023.

47. Zhao J, et al. FoxO3 coordinately activates protein degradation by the autophagic/lysosomal and proteasomal pathways in atrophying muscle cells. Cell Metab. 2007;6(6):472-483.

48. Riehle C, Abel ED. PGC-1 proteins and heart failure. Trends Cardiovasc Med. 2012;22(4):98-105.

49. Yuan J, et al. The MAPK and AMPK signalings: interplay and implication in targeted cancer therapy. J Hematol Oncol. 2020;13(1):113.

50. Verjans R, et al. MicroRNA-221/222 family counteracts myocardial fibrosis in pressure overload-induced heart failure. Hypertens. 2018;71(2):280-288.

51. Liu X, et al. miR-222 is necessary for exerciseinduced cardiac growth and protects against pathological cardiac remodeling. Cell Metab. 2015;21(4):584-95.

52. Su M, et al. MicroRNA-221 inhibits autophagy and promotes heart failure by modulating the $\mathrm{p} 27 / \mathrm{CDK} 2 / \mathrm{mTOR}$ axis. Cell Death Differ. 2015;22(6):986-999.

53. Su M, et al. Cardiac-specific overexpression of miR-222 induces heart failure and inhibits autophagy in mice. Cell Physiol Biochem. 2016;39(4):1503-1511.

54. Roux J, et al. Comparative analysis of human and mouse expression data illuminates tissue- specific evolutionary patterns of miRNAs. Nucleic Acids Res. 2012;40(13):5890-900.

55. Lagouge M, et al. Resveratrol improves mitochondrial function and protects against metabolic disease by activating SIRT1 and PGC-1alpha. Cell. 2006;127(6):1109-1122.

56. Pengo N, et al. A reversible phospho-switch mediated by ULK1 regulates the activity of autophagy protease ATG4B. Nat Commun. 2017;8(1):294.

57. Wang B, et al. AMPK $\alpha 2$ protects against the development of heart failure by enhancing mitophagy via PINK1 phosphorylation. Circ Res. 2018;122(5):712-729.

58. Drake JC, et al. Mitochondria-localized AMPK responds to local energetics and contributes to exercise and energetic stress-induced mitophagy. Proc Natl Acad Sci US A. 2021;118(37):e2025932118.

59. Song J, et al. Quality control of the mitochondrial proteome. Nat Rev Mol Cell Biol. 2021;22(1):54-70.

60. Zhu $\mathrm{H}$, et al. Mitochondrial quality surveillance as a therapeutic target in myocardial infarction. Acta Physiol (Oxf). 2021;231(3):e13590.

61. Ishizawa J, et al. Mitochondrial ClpP-mediated proteolysis induces selective cancer cell lethality. Cancer Cell. 2019;35(5):721-737.

62. Kasashima K, et al. Maintenance of mitochondrial genome distribution by mitochondrial AAA+ protein ClpX. Exp Cell Res. 2012;318(18):2335-2343.

63. Castellano E, Santos E. Functional specificity of ras isoforms: so similar but so different. Genes Cancer. 2011;2(3):216-231.

64. Esteban LM, et al. Targeted genomic disruption of $\mathrm{H}$-ras and $\mathrm{N}$-ras, individually or in combination, reveals the dispensability of both loci for mouse growth and development. Mol Cell Biol. 2001;21(5):1444-1452.

65. Newlaczyl AU, et al. Quantification of spatiotemporal patterns of Ras isoform expression during development. Sci Rep. 2017;7:41297.

66. Ulgherait M, et al. AMPK modulates tissue and organismal aging in a non-cell-autonomous manner. Cell Rep. 2014;8(6):1767-1780.

67. Viollet B, et al. AMPK: lessons from transgenic and knockout animals. Front Biosci. 2009;14(1):19-44

68. Cantó C, et al. Interdependence of AMPK and SIRT1 for metabolic adaptation to fasting and exercise in skeletal muscle. Cell Metab. 2010;11(3):213-219.

69. Danhelovska T, et al. Multisystem mitochondrial diseases due to mutations in mtDNAencoded subunits of complex I. BMC Pediatr. 2020;20(1):41.

70. Fukuda R, et al. Stimulation of glycolysis promotes cardiomyocyte proliferation after injury in adult zebrafish. EMBO Rep. 2020;21(8):e49752.

71. Tran DH, Wang ZV. Glucose metabolism in cardiac hypertrophy and heart failure. J Am Heart Assoc. 2019;8(12):e012673.

72. Morice-Picard F, et al. Cutaneous manifestations in Costello and cardiofaciocutaneous syndrome: report of 18 cases and literature review. Pediatr
Dermatol. 2013;30(6):665-673.

73. Zhang $P$, et al. AMP activated protein kinase- $\alpha 2$ deficiency exacerbates pressure-overloadinduced left ventricular hypertrophy and dysfunction in mice. Hypertension. 2008;52(5):918-924.

74. Ikeda Y, et al. Cardiac-specific deletion of LKB1 leads to hypertrophy and dysfunction. J Biol Chem. 2009;284(51):35839-35849.

75. Greer EL, et al. The energy sensor AMP-activated protein kinase directly regulates the mammalian FOXO3 transcription factor. J Biol Chem. 2007;282(41):30107-30119.

76. Johri A, et al. Pharmacologic activation of mitochondrial biogenesis exerts widespread beneficial effects in a transgenic mouse model of Huntington's disease. Hum Mol Genet. 2012;21(5):1124-1137.

77. Dumont M, et al. Bezafibrate administration improves behavioral deficits and tau pathology in P301S mice. Hum Mol Genet. 2012;21(23):5091-5105.

78. Viscomi C, et al. In vivo correction of COX deficiency by activation of the AMPK/PGC- $1 \alpha$ axis. Cell Metab. 2011;14(1):80-90.

79. Dillon LM, et al. Long-term bezafibrate treatment improves skin and spleen phenotypes of the mtDNA mutator mouse. PLoS One. 2012;7(9):e44335.

80. Yatsuga S, Suomalainen A. Effect of bezafibrate treatment on late-onset mitochondrial myopathy in mice. Hum Mol Genet. 2012;21(3):526-535.

81. Nakajima T, et al. Bezafibrate at clinically relevant doses decreases serum/liver triglycerides via down-regulation of sterol regulatory element-binding protein-1c in mice: a novel peroxisome proliferator-activated receptor alpha-independent mechanism. Mol Pharmacol. 2009;75(4):782-792.

82. Jonkers IJ, et al. Bezafibrate reduces heart rate and blood pressure in patients with hypertriglyceridemia. J Hypertens. 2001;19(4):749-755.

83. Bonnefont JP, et al. Long-term follow-up of bezafibrate treatment in patients with the myopathic form of carnitine palmitoyltransferase 2 deficiency. Clin Pharmacol Ther. 2010;88(1):101-108.

84. Steele H, et al. Metabolic effects of bezafibrate in mitochondrial disease. $E M B O \mathrm{Mol}$ Med. 2020;12(3):e11589.

85. Liu S, et al. Effect of urolithin A supplementation on muscle endurance and mitochondrial health in older adults: a randomized clinical trial. JAMA Netw Open . 2022;5(1):e2144279.

86. Andreux PA, et al. The mitophagy activator urolithin A is safe and induces a molecular signature of improved mitochondrial and cellular health in humans. Nat Metab. 2019;1(6):595-603.

87. Ryu D, et al. Urolithin A induces mitophagy and prolongs lifespan in C. elegans and increases muscle function in rodents. Nat Med. 2016;22(8):879-888.

88. Liu J, et al. AMPK: a balancer of the renin-angiotensin system. Biosci Rep. 2019;39(9):BSR20181994. 